\title{
ON THE DE RHAM-WITT COMPLEX IN MIXED CHARACTERISTIC
}

\author{
By LARS HESSELHOLT ${ }^{1}$ AND IB MADSEN ${ }^{2}$
}

ABSTRACT. - The purpose of this paper is twofold. Firstly, it gives a thorough treatment of the de RhamWitt complex for $\mathbb{Z}_{(p)}$-algebras, a construction we first considered in [L. Hesselholt, I. Madsen, Ann. of Math. 158 (2003) 1-113]. This complex is the natural generalization to $\mathbb{Z}_{(p)}$-algebras of the de Rham-Witt complex for $\mathbb{F}_{p}$-algebras of Bloch-Deligne-Illusie [L. Illusie, Ann. Sci. École Norm. Sup. 12 (4) (1979) 501-661] (for $p$ odd). We also give an explicit formula for the de Rham-Witt complex of a polynomial ring in terms of that of the coefficient ring. Secondly, we generalize the main Theorem C of [L. Hesselholt, I. Madsen, Ann. of Math. 158 (2003) 1-113] to smooth algebras over a discrete valuation ring of mixed characteristic $(0, p)$ with perfect residue field and $p$ odd.

(C) 2004 Elsevier SAS

RÉSUMÉ. - Le but de cet article est double. D'abord, il donne un traitement complet du complexe de de Rham-Witt pour les $\mathbb{Z}_{(p)}$-algèbres, une construction que nous avons considérée précédemment dans [L. Hesselholt, I. Madsen, Ann. of Math. 158 (2003) 1-113]. Ce complexe est la généralisation naturelle aux $\mathbb{Z}_{(p)}$-algèbres du complexe de de Rham-Witt pour les $\mathbb{F}_{p}$-algèbres de Bloch-Deligne-Illusie [L. Illusie, Ann. Sci. École Norm. Sup. 12 (4) (1979) 501-661] (pour $p$ impair). Nous donnons aussi une formule explicite pour le complexe de de Rham-Witt d'un anneau polynomial en termes de celui de l'anneau des coefficients. Ensuite, nous généralisons le théorème principal de [L. Hesselholt, I. Madsen, Ann. of Math. 158 (2003) 1-113] (Theorem C) aux algèbres lisses sur un anneau de valuation discret de non égale caractéristique $(0, p)$ avec corps résiduel parfait et $p$ impair.

(c) 2004 Elsevier SAS

\section{Introduction}

For every ring $A$, the cyclotomic trace is a map of pro-abelian groups

$$
\operatorname{tr}: K_{q}(A) \rightarrow \mathrm{TC}_{q}(A ; p)
$$

from the algebraic $K$-theory of $A$ to the topological cyclic homology of $A$ [2]. This is a highly non-trivial invariant. For instance, it induces an isomorphism with $\mathbb{Z} / p^{v}$-coefficients in nonnegative degrees, if $A$ is a finite algebra over the ring $W(k)$ of Witt vectors of a perfect field of characteristic $p>0$. There is a natural long-exact sequence

$$
\cdots \rightarrow \mathrm{TC}_{q}(A ; p) \rightarrow \mathrm{TR}_{q}(A ; p) \stackrel{1-F}{\longrightarrow} \mathrm{TR}_{q}(A ; p) \rightarrow \mathrm{TC}_{q-1}(A ; p) \rightarrow \cdots
$$

\footnotetext{
${ }^{1}$ The author was supported in part by a grant from the National Science Foundation.

2 The author was supported in part by the American Institute of Mathematics. 
and it is the pro-groups $\operatorname{TR}_{q}(A ; p)$ which are our main object of study here. We recall from [8, Theorem A] that the limit $\operatorname{TR}_{q}(A ; p)$ coincides with the $p$-typical curves on $K_{q+1}(A)$ introduced by Bloch in [1]. Here and throughout we assume that $A$ is a $\mathbb{Z}_{(p)}$-algebra with $p$ an odd prime.

Associated with the ring $A$, one has the topological Hochschild spectrum $T(A)$. It has an action by the circle group $\mathbb{T}$, and by definition

$$
\operatorname{TR}_{q}^{n}(A ; p)=\pi_{q}\left(T(A)^{C_{p^{n-1}}}\right)
$$

is the $q$ th homotopy group of the fixed points by the finite subgroup of the indicated order. Usually these are very large abelian groups. But they are, as $n$ and $q$ varies, related by a number of operators, and the combined algebraic structure is quite rigid. We call this structure a Witt complex over $A$. By definition, this is:

(i) a pro-differential graded ring $E^{*}$ and a strict map of pro-rings

$$
\lambda: W .(A) \rightarrow E^{0}
$$

from the pro-ring of Witt vectors in $A$;

(ii) a strict map of pro-graded rings

$$
F: E^{*} \rightarrow E_{\cdot-1}^{*}
$$

such that $\lambda F=F \lambda$ and such that for all $a \in A$,

$$
F d \lambda\left([a]_{n}\right)=\lambda\left([a]_{n-1}\right)^{p-1} d \lambda\left([a]_{n-1}\right)
$$

where $[a]_{n}=(a, 0, \ldots, 0) \in W_{n}(A)$ is the multiplicative representative;

(iii) a strict map of graded $E^{*}$-modules

$$
V: F_{*} E_{\cdot-1}^{*} \rightarrow E^{*}
$$

such that $\lambda V=V \lambda$ and such that

$$
F d V=d, \quad F V=p
$$

A map of Witt complexes over $A$ is a strict map $f: E^{*} \rightarrow E^{\prime *}$ of pro-differential graded rings such that $\lambda^{\prime}=f \lambda, F^{\prime} f=f F$ and $V^{\prime} f=f V$.

In the Witt complex $E^{*}=\mathrm{TR}_{*}(A ; p)$, the map $F$ is induced from the obvious inclusion, $V$ is the accompanying transfer map, and the differential is induced from the $\mathbb{T}$-action. The structure maps in the pro-system and the map $\lambda$ are harder to define. The map $\lambda$ turns out to be an isomorphism in this case [10, Theorem F]. We write $\mathcal{W}_{A}$ for the category of Witt complexes over $A$. Using standard category theory, we show:

THEOREM A. - The category $\mathcal{W}_{A}$ has an initial object $W . \Omega_{A}^{*}$. Moreover, the canonical map $\pi .: \Omega_{W .(A)}^{*} \rightarrow W . \Omega_{A}^{*}$ is surjective.

For a ring homomorphism $f: A \rightarrow A^{\prime}$, we have the direct image functor

$$
f_{*}: \mathcal{W}_{A^{\prime}} \rightarrow \mathcal{W}_{A}
$$


given by viewing a Witt complex over $A^{\prime}$ as a Witt complex over $A$ by replacing the map $\lambda$ by the composite $\lambda W .(f)$. We show that this functor has a left adjoint

$$
f^{*}: \mathcal{W}_{A} \rightarrow \mathcal{W}_{A^{\prime}}
$$

the inverse image functor. The universal properties imply that the canonical map

$$
W . \Omega_{A^{\prime}}^{*} \rightarrow f^{*} W . \Omega_{A}^{*}
$$

is an isomorphism. The proof of the existence of $f^{*}$, again, is by category theory. However, in the case of the ring homomorphism

$$
\pi: A \rightarrow A[x]
$$

given by the inclusion of the constant polynomials, we can give an explicit description of the inverse image functor. If $E=E^{*}$ is a Witt complex over $A$, we consider the pro-graded abelian group

$$
P(E)=P(E)^{*}
$$

where $P(E)_{n}^{q}$ is given by the set of (finite) formal sums of the form

$$
\sum_{j \in \mathbb{N}_{0}} a_{0, j}^{(n)}[x]_{n}^{j}+\sum_{j \in \mathbb{N}} b_{0, j}^{(n)}[x]_{n}^{j-1} d[x]_{n}+\sum_{s=1}^{n-1} \sum_{j \in I_{p}}\left(V^{s}\left(a_{s, j}^{(n-s)}[x]_{n-s}^{j}\right)+d V^{s}\left(b_{s, j}^{(n-s)}[x]_{n-s}^{j}\right)\right),
$$

with the components $a_{s, j}^{(m)} \in E_{m}^{q}$ and $b_{s, j}^{(m)} \in E_{m}^{q-1}$ and with $[x]_{n}$ a formal variable of degree 0 . Addition is component-wise, and the structure maps in the pro-system are induced from the ones in $E$. If $E^{\prime}=E^{\prime *}$ is a Witt complex over $A[x]$ and if $f: E \rightarrow \pi_{*} E^{\prime}$ is a map of Witt complexes over $A$, there is an induced map of pro-graded abelian groups

$$
\tilde{f}: P(E) \rightarrow E^{\prime}
$$

which maps the formal sum above to the sum

$$
\begin{aligned}
& \sum_{j \in \mathbb{N}_{0}} f\left(a_{0, j}^{(n)}\right) \lambda^{\prime}\left([x]_{n}^{j}\right)+\sum_{j \in \mathbb{N}} f\left(b_{0, j}^{(n)}\right) \lambda^{\prime}\left([x]_{n}^{j-1}\right) d \lambda^{\prime}\left([x]_{n}\right) \\
& \quad+\sum_{s=1}^{n-1} \sum_{j \in I_{p}}\left(V^{s}\left(f\left(a_{s, j}^{(n-s)}\right) \lambda^{\prime}\left([x]_{n-s}^{j}\right)\right)+d V^{s}\left(f\left(b_{s, j}^{(n-s)}\right) \lambda^{\prime}\left([x]_{n-s}^{j}\right)\right)\right)
\end{aligned}
$$

in $E_{q}^{\prime n}$. The requirement that for all $E^{\prime}$ in $\mathcal{W}_{A^{\prime}}$, this be a map of Witt complexes leaves only one possible way to define a product, a differential, and the maps $F$ and $V$ on $P(E)$. The explicit formulas are given in Section 4.2 below.

The construction $P(E)$ may be explained as follows: The first two summands in the formula above form the sub-pro-differential graded ring

$$
E_{.}^{*} \otimes_{\mathbb{Z}_{(p)}} \Omega_{\mathbb{Z}_{(p)}^{*}[x]} \subset P(E)^{*},
$$

the Frobenius on $P(E)$ induces the map of pro-graded rings

$$
F=F \otimes F: E^{*} \otimes_{\mathbb{Z}_{(p)}} \Omega_{\mathbb{Z}_{(p)}[x]}^{*} \rightarrow E^{*} \otimes_{\mathbb{Z}_{(p)}} \Omega_{\mathbb{Z}_{(p)}[x]}^{*},
$$


given on the second factor by $F\left([x]_{n}\right)=[x]_{n-1}^{p}$ and $F d[x]_{n}=[x]_{n-1}^{p-1} d[x]_{n-1}$, and the Verschiebung on $P(E)$ induces the (partially defined) map of pro-abelian groups

$$
V=V \otimes F^{-1}: E^{*} \otimes_{\mathbb{Z}_{(p)}} F\left(\Omega_{\mathbb{Z}_{(p)}[x]}^{*}\right) \rightarrow E^{*} \otimes_{\mathbb{Z}_{(p)}} \Omega_{\mathbb{Z}_{(p)}^{*}[x]}^{*}
$$

From this point of view, $P(E)$ is the minimal extension of $E_{.}^{*} \otimes \Omega_{\mathbb{Z}_{(p)}[x]}^{*}$ that admits a globally defined Verschiebung operator.

TheOREM B. - Let E be a Witt complex over A. Then $P(E)$ is a Witt complex over $A[x]$, and the canonical map

$$
\pi^{*} E \rightarrow P(E)
$$

is an isomorphism.

This gives, in particular, the promised formula for the de Rham-Witt complex of $A[x]$ in terms of that of $A$. Indeed, the canonical map

$$
P\left(W . \Omega_{A}^{*}\right) \rightarrow W . \Omega_{A[x]}^{*}
$$

is the inverse isomorphism. We also show:

THEOREM C. - The canonical map

$$
P\left(\mathrm{TR}_{*}(A ; p)\right) \rightarrow \mathrm{TR}_{*}(A[x] ; p)
$$

is an isomorphism.

The construction given in [13] of the de Rham-Witt complex for $\mathbb{F}_{p}$-algebras proceeds in two steps. Firstly, one considers a category $\mathcal{W}_{A}^{\prime}$ (denoted $\operatorname{VDR}(A)$ in op. cit.) whose objects in essence are Witt complexes without an $F$-operator. This category has an initial object $W^{\prime} \Omega_{A}^{*}$, which can be constructed somewhat more concretely. This works for all rings. Secondly, one constructs an $F$-operator on $W^{\prime} \Omega_{A}^{*}$ and proves that the combined structure is a Witt complex, which then necessarily is the initial object of $\mathcal{W}_{A}$. The proof given in op. cit. works only for $\mathbb{F}_{p}$-algebras. For it uses that for a polynomial algebra over $\mathbb{F}_{p}$, the inverse limit $W^{\prime} \Omega_{A}=\lim _{n} W_{n}^{\prime} \Omega_{A}^{*}$ is torsion free, and this is not the case for a polynomial algebra over $\mathbb{Z}_{(p)}$. We give a different proof based on Theorem B. Hence, for every $\mathbb{Z}_{(p)}$-algebra we have:

THEOREM D. - The forgetful functor $\mathcal{W}_{A} \rightarrow \mathcal{W}_{A}^{\prime}$ preserves initial objects.

Let $V$ be a complete discrete valuation ring of mixed characteristic $(0, p)$ with quotient field $K$ and perfect residue field $k$. Our second objective in this paper is to generalize [9, Theorem C] to smooth $V$-algebras. To state the result, we first recall the notion of a log-differential graded ring from [14].

A log-ring $(R, M)$ is a ring $R$ together with a pre-log structure defined as a map of multiplicative monoids $\alpha: M \rightarrow R$, and a log-differential graded ring $(D, M)$ is a differential graded ring $D$, a pre-log structure $\alpha: M \rightarrow D^{0}$, and a map of monoids $D \log : M \rightarrow\left(D^{1},+\right)$ such that $d \alpha(a)=\alpha(a) D \log a$, for all $a \in M$. We note that a pre-log structure on $R$ induces one on $W_{n}(R)$ by composing with the multiplicative map [ $]_{n}: R \rightarrow W_{n}(R)$. The notion of a Witt complex and Theorem A above generalize to log-rings; see [9, §3] for details. The universal example is denoted $W . \Omega_{(R, M)}^{*}$. It generalizes the construction of Hyodo-Kato [12] for $\log -\mathbb{F}_{p}$-algebras. 
Let $A$ be a smooth $V$-algebra, let $A_{k}=A \otimes_{V} k$, and let $A_{K}=A \otimes_{V} K$. The canonical logstructure on $A$ is given by the inclusion

$$
\alpha: M_{A}=A \cap A_{K}^{*} \hookrightarrow A .
$$

In this situation, one has the localization sequence in $K$-theory,

$$
\cdots \rightarrow K_{q}\left(A_{k}\right) \stackrel{i^{!}}{\rightarrow} K_{q}(A) \stackrel{j_{*}}{\rightarrow} K_{q}\left(A_{K}\right) \stackrel{\partial}{\rightarrow} K_{q-1}\left(A_{k}\right) \rightarrow \cdots .
$$

We constructed in $[9, \S 1]$ a corresponding sequence

$$
\cdots \rightarrow \mathrm{TR}_{q}\left(A_{k} ; p\right) \stackrel{i^{!}}{\rightarrow} \mathrm{TR}_{q}(A ; p) \stackrel{j_{*}}{\rightarrow} \mathrm{TR}_{q}\left(A \mid A_{K} ; p\right) \stackrel{\partial}{\rightarrow} \mathrm{TR}_{q-1}\left(A_{k} ; p\right) \rightarrow \cdots
$$

and a trace map from the sequence above. The groups $\operatorname{TR}_{*}\left(A \mid A_{K} ; p\right)$ form a Witt complex over the log-ring $\left(A, M_{A}\right)$ with the map

$$
d \log _{n}: M_{A} \rightarrow \mathrm{TR}_{1}^{n}\left(A \mid A_{K} ; p\right)
$$

given by the composite

$$
M_{A}=A \cap A_{K}^{*} \hookrightarrow A_{K}^{*} \rightarrow K_{1}\left(A_{K}\right) \stackrel{\operatorname{tr}}{\rightarrow} \operatorname{TR}_{1}^{n}\left(A \mid A_{K} ; p\right) .
$$

Hence, we have the canonical map from the universal Witt complex,

$$
W . \Omega_{\left(A, M_{A}\right)}^{*} \rightarrow \mathrm{TR}_{*}\left(A \mid A_{K} ; p\right) .
$$

If we assume that $\mu_{p^{v}} \subset K$, there is, in addition, a unique ring homomorphism

$$
S_{\mathbb{Z} / p^{v}}\left(\mu_{p^{v}}\right) \rightarrow \operatorname{TR}_{*}^{*}\left(A \mid A_{K} ; p, \mathbb{Z} / p^{v}\right),
$$

which takes a generator $\zeta \in \mu_{p^{v}}$ to the image by the trace map of the corresponding Bott element $b_{\zeta} \in K_{2}\left(K, \mathbb{Z} / p^{v}\right)$. In all we have a map of Witt complexes

$$
W . \Omega_{\left(A, M_{A}\right)}^{*} \otimes_{\mathbb{Z}} S_{\mathbb{Z} / p^{v}}\left(\mu_{p^{v}}\right) \rightarrow \mathrm{TR}_{*}\left(A \mid A_{K} ; p, \mathbb{Z} / p^{v}\right),
$$

where on the left, the maps $R, F$, and $V$ act as the identity on the second tensor factor. The differential acts trivially on the second tensor factor.

THEOREM E. - Let $V$ be a discrete valuation ring of mixed characteristic $(0, p)$ with quotient field $K$ and perfect residue field $k$, and assume that $p$ is odd and that $\mu_{p^{v}} \subset K$. Then for every smooth $V$-algebra A, the canonical map

$$
W \cdot \Omega_{\left(A, M_{A}\right)}^{*} \otimes_{\mathbb{Z}} S_{\mathbb{Z} / p^{v}}\left(\mu_{p^{v}}\right) \rightarrow \mathrm{TR}_{*}\left(A \mid A_{K} ; p, \mathbb{Z} / p^{v}\right)
$$

is an isomorphism of pro-abelian groups.

It appears an interesting problem to formulate and prove the analog of Theorem $\mathrm{E}$ for $p=2$. In this case, the right hand side of the statement is not a Witt complex over $A$ with the definition given here. For $(d \circ d)(x)=\eta \cdot d(x)$, where $\eta=\operatorname{tr}(-1)=d \log$. $(-1)$. This class is non-zero, for instance, if $A=\mathbb{Z}_{(2)}$, but the square $\eta^{2}$ is always zero, see Rognes [24, Theorem 1.5]. 
Finally, we mention that at the same time as this paper was written, A. Langer and T. Zink introduced a relative version of the de Rham-Witt complex [15], which to a map of $\mathbb{Z}_{(p)}$-algebras $R \rightarrow A$ associates a Witt complex $W . \Omega_{A / R}^{*}$. Hence, there is a canonical map $W . \Omega_{A}^{*} \rightarrow W . \Omega_{A / R}^{*}$. This map is always surjective, but it is not injective if $R=\mathbb{Z}_{(p)}$. The elements of the kernel are important for the relation to $K$-theory. For example, we show in Example 1.2.5 below that the map

$$
d \log : \mathbb{Z}_{p}^{*} / \mathbb{Z}_{p}^{* p^{v}} \rightarrow W \cdot \Omega_{\mathbb{Z}_{p}}^{1} / p^{v} W \cdot \Omega_{\mathbb{Z}_{p}}^{1}
$$

is an isomorphism of the domain onto the sub-pro-abelian group of the target fixed by the Frobenius operator. It takes the class of $\exp (p /(p-1))$, which generates the domain, to the class of $\sum_{s \geqslant 1} d V^{s}(1)$. But $W \cdot \Omega_{\mathbb{Z}_{p} / \mathbb{Z}_{(p)}}^{1} / p^{v} W \cdot \Omega_{\mathbb{Z}_{p} / \mathbb{Z}_{(p)}}^{1}$, in comparison, is zero. See also [4].

Unless otherwise stated, all rings considered in this paper will be commutative and unital $\mathbb{Z}_{(p)}$-algebras with $p$ an odd prime. We denote by $\mathbb{N}$ (respectively by $\mathbb{N}_{0}$, respectively by $I_{p}$ ) the set of positive integers (respectively non-negative integers, respectively positive integers prime to $p$ ). By a pro-object of a category $\mathcal{C}$ we mean a functor from $\mathbb{N}$, viewed as a category with one arrow from $n+1$ to $n$, to $\mathcal{C}$, and by a strict map between pro-objects we mean a natural transformation. A general map between pro-objects $X$ and $Y$ of $\mathcal{C}$ is an element of

$$
\operatorname{Hom}_{\text {pro }-\mathcal{C}}(X, Y)=\lim _{n} \operatorname{colim}_{m} \operatorname{Hom}_{\mathcal{C}}\left(X_{m}, Y_{n}\right) \text {. }
$$

We view objects of $\mathcal{C}$ as constant pro-objects of $\mathcal{C}$.

\section{Witt complexes}

1.1. We briefly recall Witt vectors and the de Rham complex. For a fuller discussion, we refer the reader to [23, Appendix] and [21], respectively.

The de Rham complex of a ring $A$ is characterized by the following universal property: given a differential graded ring $E^{*}$ and a ring homomorphism $\lambda: A \rightarrow E^{0}$, there exists a unique map of differential graded rings

$$
\Omega_{A}^{*} \rightarrow E^{*},
$$

which in degree zero is given by the map $\lambda$. It is also easy to construct. Let $I$ be the kernel of the multiplication $A \otimes A \rightarrow A$. It is generated as an $A$-module by the elements $a \otimes 1-1 \otimes a, a \in A$. The two $A$-module structures on $I$ define the same $A$-module structure on $\Omega_{A}^{1}=I / I^{2}$, and the map

$$
d: A \rightarrow \Omega_{A}^{1}
$$

which takes $a$ to $(a \otimes 1-1 \otimes a)+I^{2}$ is a derivation. This is the universal derivation from $A$ to an $A$-module. One now defines the de Rham-complex to be the exterior algebra

$$
\Omega_{A}^{*}=\Lambda_{A}^{*} \Omega_{A}^{1}
$$

with differential

$$
d\left(a_{0} d a_{1} \ldots d a_{n}\right)=d a_{0} d a_{1} \ldots d a_{n} .
$$

It is a differential graded ring and clearly has the universal property stated above.

The ring $W_{n}(A)$ of Witt vectors of length $n$ in $A$ is the set of $n$-tuples in $A$ but with a new ring structure characterized by the requirement that the "ghost" map

$$
w: W_{n}(A) \rightarrow A^{n}
$$


which takes the vector $\left(a_{0}, a_{1}, \ldots, a_{n-1}\right)$ to the sequence $\left(w_{0}, w_{1}, \ldots, w_{n-1}\right)$ with

$$
w_{i}=a_{0}^{p^{i}}+p a_{1}^{p^{i-1}}+\cdots+p^{i} a_{i}
$$

be a natural transformation of functors from rings to rings. If the ring $A$ is $p$-torsion free, the ghost map is injective. If, in addition, there exists a ring homomorphism $f: A \rightarrow A$ with the property that $f(a) \equiv a^{p}$ modulo $p A$, then a sequence $\left(x_{0}, \ldots, x_{n-1}\right)$ is in the image of the ghost map if and only if

$$
x_{i} \equiv f\left(x_{i-1}\right) \quad \text { modulo } p^{i} A,
$$

for all $0<i<n$. The latter statement, the lemma of Dwork, encodes the congruences needed to construct every map involving Witt vectors. As an example of how this works, we construct the addition on $W_{n}(A)$.

By naturality, it suffices to consider $A=\mathbb{Z}\left[a_{0}, \ldots, a_{n-1}, b_{0}, \ldots, b_{n-1}\right]$ and define the sum of the vectors $a=\left(a_{0}, \ldots, a_{n-1}\right)$ and $b=\left(b_{0}, \ldots, b_{n-1}\right)$. The ring homomorphism $f: A \rightarrow A$, which raises the variables to the $p$ th power, is a lift of the Frobenius, so we can use the lemma of Dwork to identify the image of the ghost map. One verifies immediately that the sequence $w(a)+w(b)$ is in the image of the ghost map. Hence, there exists a vector $s=\left(s_{0}, \ldots, s_{n-1}\right)$ such that

$$
w\left(s_{0}, \ldots, s_{n-1}\right)=w\left(a_{0}, \ldots, a_{n-1}\right)+w\left(b_{0}, \ldots, b_{n-1}\right),
$$

and since $A$ is $p$-torsion free, the vector $s$ is unique. The only possible definition, therefore, is that $a+b=s$.

The projection on the first $n-1$ factors is a ring homomorphism

$$
R: W_{n}(A) \rightarrow W_{n-1}(A)
$$

called restriction, and this makes $W .(A)$ a pro-ring. There is a second ring homomorphism, the Frobenius,

$$
F: W_{n}(A) \rightarrow W_{n-1}(A)
$$

characterized by the formula

$$
w\left(F\left(a_{0}, \ldots, a_{n-1}\right)\right)=\left(w_{1}(a), \ldots, w_{n-1}(a)\right),
$$

and a $W_{n}(A)$-linear map, the Verschiebung,

$$
V: F_{*} W_{n-1}(A) \rightarrow W_{n}(A)
$$

given by

$$
V\left(a_{0}, \ldots, a_{n-2}\right)=\left(0, a_{0}, \ldots, a_{n-1}\right) .
$$

Here the notation $F_{*} W_{n-1}(A)$ indicates that $W_{n-1}(A)$ is considered a $W_{n}(A)$-module via the Frobenius $F: W_{n}(A) \rightarrow W_{n-1}(A)$. Thus, the linearity of the Verschiebung is the statement that for all $x \in W_{n}(A)$ and $y \in W_{n-1}(A)$, the Frobenius reciprocity formula $x V(y)=V(F(x) y)$ holds. The Frobenius and Verschiebung both commute with the restriction. The Teichmüller map is the multiplicative map

$$
[]_{n}: A \rightarrow W_{n}(A)
$$


given by $[a]_{n}=(a, 0, \ldots, 0)$. In particular, $[1]_{n}$ is the multiplicative unit in $W_{n}(A)$. The following relations hold

$$
F\left([a]_{n}\right)=[a]_{n-1}^{p}, \quad F V=p,
$$

where on the right, $p$ denotes multiplication by $p=[1]+\cdots+[1]$ ( $p$ times). In general, it is very difficult to describe the coordinates of the vector $p \cdot a$ in terms of the coordinates of $a$. It is often convenient to display a Witt vector as

$$
\left(a_{0}, \ldots, a_{n-1}\right)=\sum_{i=0}^{n-1} V^{i}\left(\left[a_{i}\right]_{n-i}\right) .
$$

1.2. The definition of a Witt complex over $A$ was given in the introduction. The following result will be used repeatedly throughout the paper.

LEMMA 1.2.1. - Let $E^{*}$ be a Witt complex over A. Then

$$
d F=p F d, \quad V d=p d V, \quad V(x d y)=V(x) d V(y) .
$$

Proof. - Let $x, y \in E_{n}^{*}$. Then

$$
\begin{aligned}
V(x d y) & =V(x F d V(y))=V(x) d V(y) \\
d F(x) & =F d V F(x)=F d(V(1) x)=F(d V(1) x+V(1) d x) \\
& =F d V(1) F(x)+F V(1) F d x=d(1) F(x)+p F d x=p F d x ; \\
V d(x) & =V(1) d V(x)=d(V(1) V(x))-d V(1) \cdot V(x) \\
& =d V(F V(1) x)-V(F d V(1) x)=d V(p x)-V(d(1) x)=p d V(x) .
\end{aligned}
$$

This completes the proof.

Proof of Theorem A. - The existence of an initial object follows from the Freyd adjoint functor theorem, $\left[18\right.$, p. 116]. The category $\mathcal{W}_{A}$ clearly has all small limits, so it suffices to verify the solution set condition. To this end, we show that for every $E=E^{*}$ in $\mathcal{W}_{A}$, the image of the map induced from $\lambda$,

$$
\lambda: \Omega_{W .(A)}^{*} \rightarrow E_{.}^{*},
$$

is a (sub) Witt complex of $E$. Since the isomorphism classes of such images form a set, the proposition will follow. We must show that the Frobenius and Verschiebung of $E^{*}$ preserve the image of the canonical map. To prove the statement for the Frobenius, it suffices, since $F$ is multiplicative, to show that for all $n \geqslant 1$ and all $a \in W_{n}(A), F d \lambda(a)$ is in the image of the canonical map. But, using the formula

$$
a=\left[a_{0}\right]_{n}+V\left(\left[a_{1}\right]_{n-1}\right)+V^{2}\left(\left[a_{2}\right]_{n-2}\right)+\cdots+V^{n-1}\left(\left[a_{n-1}\right]_{1}\right),
$$

we find

$$
F d \lambda(a)=\lambda\left(\left[a_{0}\right]_{n-1}\right)^{p-1} d \lambda\left(\left[a_{0}\right]_{n-1}\right)+d \lambda\left(\left[a_{1}\right]_{n-1}\right)+\cdots+d V^{n-1} \lambda\left(\left[a_{n-1}\right]_{1}\right),
$$

and this sum clearly is in the image of the canonical map. The statement for the Verschiebung follows immediately from Lemma 1.2.1. This proves that an initial object exists. 
Finally, we show that the map $\pi$. is surjective, or equivalently, that the inclusion of the image $E$ of this map is a surjection. Since $E$ is a Witt complex, there is a unique map $W . \Omega_{A}^{*} \rightarrow E^{*}$ of Witt complexes. But then also the composition

$$
W . \Omega_{A}^{*} \rightarrow E^{*} \rightarrow W . \Omega_{A}^{*}
$$

is a map of Witt complexes. And since $W . \Omega_{A}^{*}$ is the initial object, this composite is the identity map. The statement follows.

Remark 1.2.2. - Theorem A shows, in particular, that the canonical map

$$
W .(A) \rightarrow W . \Omega_{A}^{0}
$$

is surjective. In effect, this is an isomorphism. For $E^{*}=W .(A)$ is a Witt complex over $A$. We will prove later that also the canonical map $\Omega_{A}^{*} \rightarrow W_{1} \Omega_{A}^{*}$ is an isomorphism. The proof of this, however, requires Theorem $\mathrm{D}$.

The direct image functor $f_{*}: \mathcal{W}_{B} \rightarrow \mathcal{W}_{A}$ associated with a ring homomorphism $f: A \rightarrow B$ takes $E_{\text {. }}^{*}$ to $E_{\text {. }}^{*}$ and replaces the map $\lambda$ by the composite $\lambda W$. $(f)$.

PROPOSITION 1.2.3. - The direct image functor $f_{*}$ has a left adjoint

$$
f^{*}: \mathcal{W}_{A} \rightarrow \mathcal{W}_{B}
$$

the inverse image functor.

Proof. - The proof, which is similar to the proof of Theorem A, is an application of the adjoint functor theorem, [18, p. 116]. Given an object $E=E^{*}$. in $\mathcal{W}_{A}$, the object $f^{*} E$ in $\mathcal{W}_{B}$ is the initial object in the over category $E / f_{*}$. This category has small limits, so we must verify the solution set condition.

We first construct, for all $n \geqslant 1$, a non-commutative graded ring $T_{n}^{*}$ which depends only on $E$. Assume, inductively, that $T_{n-1}^{*}$ has been constructed (we let $T_{0}^{*}=\{0\}$ ), and let

$$
S_{n}^{*}=\left\{e, d e \mid e \in W_{n}(B) \otimes_{W_{n}(A)} E_{n}^{*}\right\} \cup\left\{V\left(e^{\prime}\right), d V\left(e^{\prime}\right) \mid e^{\prime} \in T_{n-1}^{*}\right\}
$$

be the graded set, where $e$ and $V\left(e^{\prime}\right)$ are assigned the degree of $e$ and $e^{\prime}$, respectively, and where the degree of $d e$ and $d V\left(e^{\prime}\right)$ is one higher than the degree of $e$ and $e^{\prime}$. Then we define $T_{n}^{*}$ to be the free non-commutative graded ring generated by the graded set of $S_{n}^{*}$.

Given an object $\left(D, \varphi: E \rightarrow f_{*} D\right)$ of the over category $E / f_{*}$, we recursively define maps of graded rings

$$
\psi_{n}: T_{n}^{*} \rightarrow D_{n}^{*}
$$

The given map of graded $W_{n}(A)$-algebras $\varphi_{n}: E_{n}^{*} \rightarrow f_{*} D_{n}^{*}$ induces a map of graded $W_{n}(B)$-algebras

$$
\hat{\varphi}_{n}: W_{n}(B) \otimes_{W_{n}(A)} E_{n}^{*} \rightarrow D_{n}^{*},
$$

and with $\psi_{n-1}: T_{n-1}^{*} \rightarrow D_{n-1}^{*}$ already defined, we let $\psi_{n}^{\prime}: S_{n}^{*} \rightarrow D_{n}^{*}$ be the map of graded sets given by $\psi_{n}(e)=\hat{\varphi}_{n}(e), \psi_{n}(d e)=d\left(\hat{\varphi}_{n}(e)\right)$, for $e \in W_{n}(B) \otimes_{W_{n}(A)} E_{n}^{*}$, and by

$$
\psi_{n}\left(V\left(e^{\prime}\right)\right)=V\left(\psi_{n-1}\left(e^{\prime}\right)\right), \quad \psi_{n}\left(d V\left(e^{\prime}\right)\right)=d V\left(\psi_{n-1}\left(e^{\prime}\right)\right),
$$


for $e^{\prime} \in T_{n-1}^{*}$. Then $\psi_{n}: T_{n} \rightarrow D_{n}$ is the unique map of graded rings which extends $\psi_{n}^{\prime}$.

One shows, as in the proof of Theorem A, that the images

$$
I_{(D, \varphi)}=\left\{\operatorname{im}\left(\psi_{n}\right)\right\}_{n \geqslant 1}
$$

form a Witt complex over $B$, and that the map $\varphi^{\prime}: E \rightarrow f_{*} I_{(D, \varphi)}$, which takes $e \in E_{n}^{d}$ to $\psi_{n}(1 \otimes e) \in I_{(D, \varphi), n}^{d}$, is a map of Witt complexes over $B$. Hence, the canonical inclusion $I_{(D, \varphi)} \rightarrow D$ defines a map

$$
\left(I_{(D, \varphi)}, \varphi^{\prime}: E \rightarrow f_{*} I_{(D, \varphi)}\right) \rightarrow\left(D, \varphi: E \rightarrow f_{*} D\right)
$$

in the over category $E / f_{*}$. Since the isomorphism classes of the objects of $E / f_{*}$ of the form $\left(I_{(D, \varphi)}, \varphi^{\prime}: E \rightarrow f_{*} I_{(D, \varphi)}\right)$ form a set, the solution set condition is satisfied.

Example 1.2.4. - We consider $W . \Omega_{\mathbb{Z}_{(p)}^{*}}^{*}$. In general, an integer invertible in $A$ is also invertible in $W_{n}(A)$, and hence, the ring $W_{n}\left(\mathbb{Z}_{(p)}\right)$ is a $\mathbb{Z}_{(p)}$-algebra. We claim that as a $\mathbb{Z}_{(p)}$-module,

$$
W_{n}\left(\mathbb{Z}_{(p)}\right)=\prod_{i=0}^{n-1} \mathbb{Z}_{(p)} \cdot V^{i}(1)
$$

with the product given by

$$
V^{i}(1) \cdot V^{j}(1)=p^{i} V^{j}(1),
$$

for $0 \leqslant i \leqslant j<n$. The first statement follows by an induction argument based on the exact sequences

$$
0 \rightarrow \mathbb{Z}_{(p)} \stackrel{V^{n-1}}{\longrightarrow} W_{n}\left(\mathbb{Z}_{(p)}\right) \stackrel{R}{\rightarrow} W_{n-1}\left(\mathbb{Z}_{(p)}\right) \rightarrow 0,
$$

and the product formula is an immediate consequence of the relations $F V=p$ and $x V(y)=V(F(x) y)$. In general, it is difficult to find the coordinates of $a \in W_{n}\left(\mathbb{Z}_{(p)}\right)$ with respect to the basis $V^{i}(1), 0 \leqslant i<n$.

We can use the canonical surjection

$$
\Omega_{W_{n}\left(\mathbb{Z}_{(p)}\right)}^{*} \rightarrow W_{n} \Omega_{\mathbb{Z}_{(p)}^{*}}^{*}
$$

to get an upper bound for the right hand side. The map is an isomorphism in degree zero, and in degree one we have the relations that for $0 \leqslant i \leqslant j<n$,

$$
\begin{aligned}
& V^{i}(1) d V^{j}(1)=V^{i}\left(F^{i} d V^{j}(1)\right)=V^{i} d V^{j-i}(1)=p^{i} d V^{j}(1), \\
& V^{j}(1) d V^{i}(1)=V^{j}\left(F^{j} d V^{i}(1)\right)=V^{j} F^{j-i} d(1)=0 .
\end{aligned}
$$

It follows that $p^{i} d V^{i}(1)$ and $d V^{i}(1) d V^{j}(1)$ are zero, for all $0 \leqslant i, j<n$. Hence $W_{n} \Omega_{\mathbb{Z}_{(p)}}^{q}$ vanishes for $q>1$, and there is canonical surjection

$$
\prod_{i=0}^{n-1} \mathbb{Z} / p^{i} \mathbb{Z} \cdot d V^{i}(1) \rightarrow W_{n} \Omega_{\mathbb{Z}_{(p)}}^{1}
$$


In fact, this is an isomorphism. To prove injectivity, it suffices to find a Witt complex $E=E^{*}$. such that the canonical map

$$
\prod_{i=0}^{n-1} \mathbb{Z} / p^{i} \mathbb{Z} \cdot d V^{i}(1) \rightarrow E_{n}^{1}
$$

is injective. We show in Proposition 2.6.1 below that $\mathrm{TR}_{*}\left(\mathbb{Z}_{(p)} ; p\right)$ is such a Witt complex.

Example 1.2.5. - We next consider $W \cdot \Omega_{\mathbb{Z}_{p}}^{*}$. One proves by induction on $n \geqslant 1$ that for all $q \geqslant 0$ and $v \geqslant 1$, the completion map induces an isomorphism

$$
W_{n} \Omega_{\mathbb{Z}_{(p)}}^{q} / p^{v} W_{n} \Omega_{\mathbb{Z}_{(p)}}^{q} \stackrel{\sim}{\rightarrow} W_{n} \Omega_{\mathbb{Z}_{p}}^{q} / p^{v} W_{n} \Omega_{\mathbb{Z}_{p}}^{q}
$$

We wish to evaluate the map

$$
d \log _{n}: \mathbb{Z}_{p}^{*} / \mathbb{Z}_{p}^{* p^{v}} \rightarrow W_{n} \Omega_{\mathbb{Z}_{p}}^{q} / p^{v} W_{n} \Omega_{\mathbb{Z}_{p}}^{q},
$$

which to the class of $x \in \mathbb{Z}_{p}^{*}$ assigns the class of $[x]_{n}^{-1} d[x]_{n} \in W_{n} \Omega_{\mathbb{Z}_{p}}^{1}$. We have

$$
[x]_{n}=x \cdot[1]_{n}+\sum_{0<s<n} p^{-s}\left(x^{p^{s}}-x^{p^{s-1}}\right) \cdot V^{s}\left([1]_{n-s}\right),
$$

where we use that the $\mathbb{Z}_{(p)}$-module structure on $W_{n} \Omega_{\mathbb{Z}_{p}}^{1}$ extends to a $\mathbb{Z}_{p}$-module structure by continuity. Indeed, as one readily verifies, the two sides of the equality have the same image by the ghost map, and the ghost map is injective. If we differentiate this formula and multiply by $[x]_{n}^{-1}=\left[x^{-1}\right]_{n}$, we find that

$$
d \log _{n} x=\sum_{0<s<n} p^{-s}\left(x^{p^{s-1}(p-1)}-1\right) \cdot d V^{s}\left([1]_{n-s}\right) .
$$

In particular, the class of $\exp (p /(p-1))$, which generates the domain of $d \log _{n}$, is mapped to the class of $\sum_{0<s<n} d V^{s}(1)$. It is not difficult from Example 1.2.4 to see that as $n \geqslant 1$ varies, the latter class generates the sub-pro-abelian group of the target of $d \log _{n}$ that is fixed by the Frobenius operator.

\section{The Witt complex $\mathrm{TR}_{*}(A ; p)$}

2.1. In this paragraph we recall the Witt complex $\operatorname{TR}_{*}(A ; p)$ associated with a ring $A$. Details may be found in [5,8-10]. See also [19].

Let $G$ be a compact Lie group. The $G$-stable category is a triangulated category and a closed symmetric monoidal category, and the two structures are compatible, [16, II.3.13]. The objects of the $G$-stable category are called $G$-spectra. A monoid for the smash product is called a ring $G$-spectrum. We denote the set of maps between two $G$-spectra $T$ and $T^{\prime}$ by $\left[T, T^{\prime}\right]_{G}$.

Associated with a pointed $G$-space $X$ one has the suspension $G$-spectrum which we denote by $\operatorname{susp}_{G}(X)$ or simply by $X$. If $V$ is an orthogonal $G$-representation, we denote by $S^{V}$ the one-point compactification. Then the suspension homomorphism

$$
\left[T, T^{\prime}\right]_{G} \stackrel{\sim}{\rightarrow}\left[T \wedge S^{V}, T^{\prime} \wedge S^{V}\right]_{G}
$$


is an isomorphism, [16, I.6.1]. Let $H \subset G$ be a closed subgroup, let $q$ be an integer, and let $T$ be a $G$-spectrum. We define the (derived) homotopy group

$$
\pi_{q}^{H}(T)=\left[G / H_{+} \wedge S^{q}, T\right]_{G},
$$

where the subscript + indicates the addition of a disjoint $G$-fixed basepoint. There is a canonical isomorphism

$$
\pi_{q}^{H}(T) \cong \pi_{q}\left(T^{H}\right)
$$

where $T^{H}$ is the $H$-fixed point $W_{G} H$-spectrum. More generally, given a pair of closed subgroups $K \subset H \subset G$ with $K$ normal in $H$, there is a canonical isomorphism

$$
\pi_{q}^{H}(T) \cong \pi_{q}^{H / K}\left(T^{K}\right)
$$

A map in the $G$-stable category is an isomorphism if and only if the induced map of homotopy groups is an isomorphism, for all $H \subset G$ and all $q$, [16, I.5.12].

Let $H \subset G$ be a closed subgroup. The diagonal map of the space $G / H$ induces a map in the $G$-stable category

$$
\Delta: G / H_{+} \rightarrow G / H_{+} \wedge G / H_{+},
$$

and if $T$ and $T^{\prime}$ are $G$-spectra, this gives rise to a pairing

$$
\pi_{q}^{H}(T) \otimes \pi_{q^{\prime}}^{H}\left(T^{\prime}\right) \rightarrow \pi_{q+q^{\prime}}^{H}\left(T \wedge T^{\prime}\right) .
$$

If $T$ is a ring $G$-spectrum, we may compose with the map of homotopy groups induced by the multiplication $\mu: T \wedge T \rightarrow T$. This way the homotopy groups $\pi_{*}^{H}(T)$ form a graded ring, and if $T$ is commutative, this graded ring is commutative in the graded sense.

Finally, we mention the Segal-tom Dieck splitting, [25, Satz 2]. If $H \subset G$ is finite and if $X$ is a pointed $G$-space, there is a canonical isomorphism

$$
\bigoplus_{(K)} \pi_{q}\left(\operatorname{susp}\left(E\left(W_{H} K\right)_{+} \wedge_{W_{H} K} X^{K}\right)\right) \stackrel{\sim}{\rightarrow} \pi_{q}^{H}\left(\operatorname{susp}_{G}(X)\right),
$$

where the sum is over conjugacy classes of subgroups of $H$, and $E\left(W_{H} K\right)$ is the universal cover of the classifying space $B\left(W_{H} K\right)$.

2.2. Let $\mathbb{T}$ be the circle group. Associated with every ring $A$ one has the topological Hochschild spectrum $T(A)$. This is a ring $\mathbb{T}$-spectrum, and by definition,

$$
\operatorname{TR}_{q}^{n}(A ; p)=\left[S^{q} \wedge \mathbb{T} / C_{p^{n-1}+}, T(A)\right]_{\mathbb{T}},
$$

where $C_{p^{n-1}} \subset \mathbb{T}$ denotes the finite subgroup of the indicated order. The maps

$$
\begin{aligned}
& F: \operatorname{TR}_{q}^{n}(A ; p) \rightarrow \operatorname{TR}_{q}^{n-1}(A ; p), \\
& V: \operatorname{TR}_{q}^{n-1}(A ; p) \rightarrow \operatorname{TR}_{q}^{n}(A ; p), \\
& d: \operatorname{TR}_{q}^{n}(A ; p) \rightarrow \operatorname{TR}_{q+1}^{n}(A ; p),
\end{aligned}
$$

which are part of the structure of a Witt complex, are induced by maps in the $\mathbb{T}$-stable category 


$$
\begin{aligned}
& f: \mathbb{T} / C_{p^{n-2}+} \rightarrow \mathbb{T} / C_{p^{n-1}+}, \\
& v: \mathbb{T} / C_{p^{n-1}+} \rightarrow \mathbb{T} / C_{p^{n-2}+}, \\
& \delta: \mathbb{T} / C_{p^{n-1}+} \wedge S^{1} \rightarrow T / C_{p^{n-1}+},
\end{aligned}
$$

the definition of which we briefly recall.

The map $f$ is induced by the canonical projection of $\mathbb{T}$-spaces, and $v$ is the corresponding transfer map defined as follows. Let $i: \mathbb{T} / C_{p^{n-2}} \hookrightarrow V$ be an embedding into an orthogonal $\mathbb{T}$-representation, and consider the product embedding $(\mathrm{pr}, i): \mathbb{T} / C_{p^{n-2}} \hookrightarrow \mathbb{T} / C_{p^{n-1}} \times V$. The normal bundle of the latter is trivial, and the linear structure on $V$ gives a preferred trivialization. Hence, by the Pontryagin-Thom construction, which collapses the complement of a tubular neighborhood to the base point, we have a map of pointed $\mathbb{T}$-spaces

$$
\mathbb{T} / C_{p^{n}}+\wedge S^{V} \rightarrow \mathbb{T} / C_{p^{n-1}}+\wedge S^{V},
$$

and (under the suspension isomorphism) this induces the map $v$. Finally, the map $\delta$ is induced from a map of pointed $\mathbb{T}$-spaces

$$
\delta: \mathbb{T} / C_{p^{n-1}+} \wedge S^{m+1} \rightarrow \mathbb{T} / C_{p^{n-1}+} \wedge S^{m} .
$$

The set of $\mathbb{T}$-homotopy classes of such maps, if $m \geqslant 2$, is a direct sum of an infinite cyclic group and a cyclic group of order 2, and the map $\delta$ is a generator of an infinite cyclic summand. The induced map on reduced homology,

$$
\tilde{H}_{m+1}\left(\mathbb{T} / C_{p^{n-1}}+\wedge S^{m+1}\right) \stackrel{\delta}{\rightarrow} \tilde{H}_{m+1}\left(\mathbb{T} / C_{p^{n-1}+} \wedge S^{m}\right),
$$

takes the generator on the left which, under the canonical isomorphism

$$
\tilde{H}_{q}\left(X_{+} \wedge S^{m}\right) \cong H_{q-m}(X),
$$

corresponds to class of the point $C_{p^{n-1}}$ in $H_{0}\left(\mathbb{T} / C_{p^{n-1}}\right)$ to the generator on the right which corresponds to the fundamental class $\left[\mathbb{T} / C_{p^{n-1}}\right] \in H_{1}\left(\mathbb{T} / C_{p^{n-1}}\right)$.

If we ignore 2-torsion, these maps satisfy the following relations

$$
\begin{array}{ll}
v f=p \cdot \mathrm{id}, & f \delta=p \delta f, \quad \delta v=p v \delta, \\
v \delta f=\delta, & \delta \delta=0,
\end{array}
$$

and hence the dual relations hold among the maps (2.2.1). Moreover, there are further relations among the maps $f, v, \delta$, and the diagonal map $\Delta$. The relations

$$
(f \wedge f) \Delta=\Delta f, \quad(f \wedge i d) \Delta v=(\mathrm{id} \wedge v) \Delta,
$$

show that $F$ is a map of graded rings, and that $V$ is a map of graded modules. And the relation

$$
\Delta \delta=(\delta \wedge \mathrm{id} \vee \operatorname{id} \wedge \delta) \tau \nabla \Delta
$$

valid up to 2-torsion, shows that $d$ is a derivation. Here $\tau$ permutes the appropriate smash factors, and $\nabla$ is the fold map. The proof of these facts may be found in [10, Lemma 3.3] and [8, 1.4.2, 1.5.1]. 
Remark 2.2.4. - Up to 2-torsion, the full subcategory of the $\mathbb{T}$-stable category with objects $\mathbb{T} / C_{p^{n-1}+} \wedge S^{q}$, where $0 \leqslant q \leqslant 2$ and $n \in \mathbb{N}$, is equal to the ringoid generated by the maps (2.2.2) subject to the relations (2.2.3). In more detail, if $m$ is the minimum of $r$ and $s$, then:

(i) The maps from $\mathbb{T} / C_{p^{s}}$ to $\mathbb{T} / C_{p^{r}}$ form a free abelian group of rank $m+1$ generated by $f^{r-i} v^{s-i}$ with $0 \leqslant i \leqslant m$.

(ii) The abelian group of maps from $\mathbb{T} / C_{p^{s}} \wedge S^{1}$ to $\mathbb{T} / C_{p^{r}}$ is, up to 2 -torsion, the sum of a free abelian group of rank $m+1$ and, for every $1 \leqslant i \leqslant m$, a copy of $\mathbb{Z} / p^{i} \mathbb{Z}$. If $r \geqslant s$ (respectively if $r \leqslant s$ ) then $f^{r-i} v^{s-i} \delta$ (respectively $\delta f^{r-i} v^{s-i}$ ) is a generator of a summand $\mathbb{Z}$, and in either case, $p^{r-m} \delta f^{r-i} v^{s-i}-p^{s-m} f^{r-i} v^{s-i} \delta$ generates a summand $\mathbb{Z} / p^{m-i} \mathbb{Z}$.

(iii) The abelian group of maps from $\mathbb{T} / C_{p^{s}} \wedge \wedge S^{2}$ to $\mathbb{T} / C_{p^{r}}$ is, up to 2 -torsion, the sum for $1 \leqslant i \leqslant m$, of a copy of $\mathbb{Z} / p^{m-i} \mathbb{Z}$ generated by $\delta f^{r-i} v^{s-i} \delta$.

(iv) If $q>0$ then every map from $\mathbb{T} / C_{p^{s}}$ to $\mathbb{T} / C_{p^{r}} \wedge S^{q}$ is zero.

This follows from the Segal-tom Dieck splitting, (2.1.1).

2.3. An isomorphism $f: G \stackrel{\sim}{\rightarrow} G^{\prime}$ of compact Lie groups induces an equivalence of categories $f^{*}$ from the $G^{\prime}$-stable category to the $G$-stable category, [16, II.1.7]. If $H \subset G$ is a closed subgroup, we let $H^{\prime} \subset G^{\prime}$ be the closed subgroup $H^{\prime}=f(H)$. Then for every closed subgroup $H \subset G$ and every integer $q$, there is a canonical isomorphism of $G$-spectra

$$
G / H_{+} \wedge S^{q} \cong f^{*}\left(G^{\prime} / H^{\prime} \wedge S^{q}\right),
$$

and this induces, for every $G^{\prime}$-spectrum $T^{\prime}$, a canonical isomorphism

$$
\pi_{q}^{H}\left(f^{*}\left(T^{\prime}\right)\right) \cong \pi_{q}^{H^{\prime}}\left(T^{\prime}\right)
$$

In the case of the circle group, we have the isomorphism

$$
\rho_{p}: \mathbb{T} \stackrel{\sim}{\rightarrow} \mathbb{T} / C_{p}
$$

given by the $p$ th root. If $T$ is a $\mathbb{T}$-spectrum, then $T^{C_{p}}$ is a $\mathbb{T} / C_{p^{-}}$-spectrum, and hence, $\rho_{p}^{*}\left(T^{C_{p}}\right)$ is a $\mathbb{T}$-spectrum. We have the canonical isomorphisms

$$
\pi_{q}^{C_{p^{n-2}}}\left(\rho_{p}^{*}\left(T^{C_{p}}\right)\right) \cong \pi_{q}^{C_{p^{n-1}} / C_{p}}\left(T^{C_{p}}\right) \cong \pi_{q}^{C_{p^{n-1}}}(T)
$$

and these are compatible with the maps $F, V$, and $d$ induced from (2.2.2).

The topological Hochschild $\mathbb{T}$-spectrum $T(A)$ is a cyclotomic spectrum in the sense of [10, Definition 2.2]. This implies that there is a map of $\mathbb{T}$-spectra

$$
r: \rho_{p}^{*}\left(T(A)^{C_{p}}\right) \rightarrow T(A) .
$$

Hence, we have the map

$$
R: \operatorname{TR}_{q}^{n}(A ; p) \rightarrow \mathrm{TR}_{q}^{n-1}(A ; p)
$$

defined as the composite

$$
\pi_{q}^{C_{p^{n-1}}}(T(A)) \cong \pi_{q}^{C_{p^{n-2}}}\left(\rho_{p}^{*}\left(T(A)^{C_{p}}\right)\right) \stackrel{r}{\rightarrow} \pi_{q}^{C_{p^{n-2}}}(T(A)),
$$

and this map commutes with the operators $F, V$, and $d$. Moreover, $r$ is a map of ring $\mathbb{T}$-spectra, and hence $R$ is a map of graded rings. 
2.4. In order to construct the $\mathbb{T}$-spectrum $T(A)$ we need a model category for the $\mathbb{T}$-stable category. The model category we use is the category of symmetric spectra in the category of orthogonal $\mathbb{T}$-spectra, [20]. This model has a closed symmetric monoidal product which induces the smash product on the $\mathbb{T}$-stable category. We first recall the topological Hochschild space $\operatorname{THH}(A)$. See $[5, \S 1]$ and $[10, \S 2]$ for more details.

If $A$ is a ring and $X$ a pointed simplicial set, the homotopy groups of the space

$$
A(X)=\left|A\{X\} / A\left\{x_{0}\right\}\right|
$$

are canonically isomorphic to the reduced singular homology groups of $|X|$ with coefficients in $A$ [22, Theorem 22.1]. Here $A\{X\}$ denotes the degree-wise free $A$-module generated by $X$. Let $S^{1}=\Delta[1] / \partial \Delta[1]$ be the standard simplicial circle and let $S^{i}$ be the smash product of $i$ copies of $S^{1}$. Then

$$
\tilde{A}_{i}=A\left(S^{i}\right)
$$

is an Eilenberg-MacLane space for $A$ concentrated in degree $i$. It has a natural $\Sigma_{i}$-action given by permuting the smash factors in $S^{i}$. Moreover, there are natural maps

$$
e: S^{i} \rightarrow \tilde{A}_{i}, \quad \mu: \tilde{A}_{i} \wedge \tilde{A}_{i^{\prime}} \rightarrow \tilde{A}_{i+i^{\prime}}
$$

which are $\Sigma_{i}$-equivariant and $\Sigma_{i} \times \Sigma_{i^{\prime}}$-equivariant, respectively. This constitutes a symmetric ring spectrum $\tilde{A}$ in the sense of [11], commutative if $A$ is. The space $\operatorname{THH}(E)$ is defined for every symmetric ring spectrum $E$.

Let $I$ be the category with objects the finite sets

$$
\underline{i}=\{1,2, \ldots, i\}, \quad i \geqslant 0,
$$

and morphisms all injective maps $(\underline{0}=\emptyset)$. It is a strict monoidal (but not symmetric monoidal) category under concatenation of sets and maps. Let $E$ be a symmetric ring spectrum and let $X$ be a pointed space. There is a functor $G_{k}(E ; X)$ from $I^{k+1}$ to pointed spaces, which on objects is given by the pointed function space

$$
G_{k}(E ; X)\left(\underline{i_{0}}, \ldots, \underline{i_{k}}\right)=F\left(S^{i_{0}} \wedge \cdots \wedge S^{i_{k}}, E_{i_{0}} \wedge \cdots \wedge E_{i_{k}} \wedge X\right) .
$$

The homotopy colimit

$$
\mathrm{THH}_{k}(E ; X)=\underset{I^{k+1}}{\operatorname{holim}} G_{k}(E ; X)
$$

is naturally the space of $k$-simplices in a cyclic space, and by definition

$$
\operatorname{THH}(E ; X)=\left|[k] \mapsto \operatorname{THH}_{k}(E ; X)\right| .
$$

This is a $\mathbb{T}$-space, $[17,7.1 .4]$.

More generally, let $(n)$ be the finite ordered set $\{1,2, \ldots, n\}$. The product category $I^{(n)}$ is a strict monoidal category under component-wise concatenation of sets and maps. (The category $I^{(0)}$ is the category with one object and one morphism.) Concatenation of sets and maps according to the ordering of $(n)$ defines a functor

$$
\sqcup_{n}: I^{(n)} \rightarrow I,
$$


but this does not preserve the monoidal structure. (The functor $\sqcup_{0}$ takes the unique object to 0 .) We let $G_{k}^{(n)}(E ; X)$ be the functor from $\left(I^{(n)}\right)^{k+1}$ to the category of pointed spaces given by

$$
G_{k}^{(n)}(E ; X)=G_{k}(E ; X) \circ\left(\sqcup_{n}\right)^{k+1},
$$

and define

$$
\operatorname{THH}_{k}^{(n)}(E ; X)=\underset{\left(I^{(n)}\right)^{k+1}}{\operatorname{holim}} G_{k}^{(n)}(E ; X) .
$$

This again is the space of $k$-simplices in a cyclic space, and we define

$$
\mathrm{THH}^{(n)}(E ; X)=\left|[k] \mapsto \mathrm{THH}_{k}^{(n)}(E ; X)\right| .
$$

It is a $\Sigma_{n} \times \mathbb{T}$-space. If $E$ is commutative, there is a natural product

$$
\mathrm{THH}^{(m)}(E ; X) \wedge \mathrm{THH}^{(n)}(E ; Y) \rightarrow \mathrm{THH}^{(m+n)}(E ; X \wedge Y),
$$

which is $\Sigma_{m} \times \Sigma_{n} \times \mathbb{T}$-equivariant with $\mathbb{T}$ acting diagonally on the left.

Let $V$ be a finite dimensional orthogonal $\mathbb{T}$-representation. We define the $(n, V)$ th space in the symmetric orthogonal $\mathbb{T}$-spectrum $T(E)$ by

$$
T(E)_{n, V}=\mathrm{THH}^{(n)}\left(E ; S^{\mathbb{R}^{n} \oplus V}\right) .
$$

There are two $\mathbb{T}$-actions on this space: one which comes from the topological Hochschild space, and another induced from the $\mathbb{T}$-action on $S^{V}$. There are also two $\Sigma_{n}$-actions: one which comes from the $\Sigma_{n}$-action on the topological Hochschild space, and another induced from the permutation representation of $\Sigma_{n}$ on $\mathbb{R}^{n}$. We give $T(E)_{n, V}$ the diagonal $\Sigma_{n} \times \mathbb{T}$-action. If $E$ is commutative, there is, in addition, a $\Sigma_{m} \times \Sigma_{n} \times \mathbb{T}$-equivariant product

$$
T(E)_{m, V} \wedge T(E)_{n, W} \rightarrow T(E)_{m+n, V \oplus W} .
$$

This product makes $T(E)$ a monoid in the symmetric monoidal category of symmetric orthogonal $\mathbb{T}$-spectra.

2.5. A pointed monoid is a monoid $\Pi$ in the category of pointed spaces and smash product. The unit and multiplication are maps

$$
e: S^{0} \rightarrow \Pi, \quad \mu: \Pi \wedge \Pi \rightarrow \Pi .
$$

The $(k+1)$-fold smash product

$$
N_{k}^{\text {cy }}(\Pi)=\Pi^{\wedge(k+1)}
$$

is the $k$-simplices of a cyclic space. The geometric realization

$$
N^{\mathrm{cy}}(\Pi)=\left|[k] \mapsto N_{k}^{\mathrm{cy}}(\Pi)\right|
$$

is a pointed $\mathbb{T}$-space called the cyclic bar construction of $\Pi$, see $[17,7.3 .10]$. It comes equipped with a natural $\mathbb{T}$-equivariant homeomorphism $[2,1.1,2.3]$

$$
\Delta: N^{\mathrm{cy}}(\Pi) \stackrel{\sim}{\rightarrow} \rho_{p^{n-1}}^{*}\left(N^{\mathrm{cy}}(\Pi)^{C_{p^{n-1}}}\right) .
$$


If $E$ is a symmetric ring spectrum, then the 0 th space $E_{0}$ is a pointed monoid. In the case $E=\tilde{A}$, this is the underlying multiplicative monoid of the ring $A$ with basepoint 0 . In the symmetric orthogonal $\mathbb{T}$-spectrum $T(E)$ defined above, the $(0,0)$ th space is

$$
T(E)_{0,0}=N^{\mathrm{cy}}\left(E_{0}\right) .
$$

Hence, there is a canonical map

$$
k: \pi_{q}\left(\rho_{p^{n-1}}^{*} N^{\mathrm{cy}}\left(E_{0}\right)^{C_{p^{n-1}}}\right) \rightarrow \pi_{q}\left(\rho_{p^{n-1}}^{*} T(E)^{C_{p^{n-1}}}\right)=\operatorname{TR}_{q}^{n}(E ; p) .
$$

We define a map of pointed sets

$$
\omega_{n}: \pi_{0}\left(E_{0}\right) \rightarrow \mathrm{TR}_{0}^{n}(E ; p)
$$

to be the composite

$$
\pi_{0}\left(E_{0}\right) \rightarrow \pi_{0}\left(N^{\mathrm{cy}}\left(E_{0}\right)\right) \stackrel{\Delta}{\rightarrow} \pi_{0}\left(\rho_{p^{n-1}}^{*} N^{\mathrm{cy}}\left(E_{0}\right)^{C_{p^{n-1}}}\right) \stackrel{k}{\rightarrow} \pi_{0}\left(\rho_{p^{n-1}}^{*} T(E)^{C_{p^{n-1}}}\right),
$$

where the left hand map is induced by the inclusion of the vertices. If $E$ is commutative, this is a multiplicative map. It is proved in [8, Lemma 1.5.6] that for every $x \in \pi_{0}\left(E_{0}\right)$,

$$
F d \omega_{n}(x)=\omega_{n-1}(x)^{p-1} d \omega_{n-1}(x) .
$$

For $E=\tilde{A}$, we now define the map

$$
\lambda: W_{n}(A) \rightarrow \mathrm{TR}_{0}^{n}(A ; p)
$$

by the formula

$$
\lambda\left(a_{0}, \ldots, a_{n}\right)=\sum_{s=0}^{n-1} V^{s}\left(\omega_{n-s}\left(a_{s}\right)\right) .
$$

It is proved in [10, Theorem F] that this is an isomorphism of rings. This completes our recollection of the Witt complex $\operatorname{TR}_{*}(A ; p)$.

2.6. Let $\mathbb{S}$ be the symmetric ring spectrum with $\mathbb{S}_{i}=S^{i}$. This is the sphere spectrum. It was proved in [2,3.7], but see also [19, 4.4.4], that the unit for the ring spectrum structure

$$
\operatorname{susp}_{\mathbb{T}}\left(S^{0}\right) \rightarrow T(\mathbb{S})
$$

induces an isomorphism of homotopy groups, for all integers $q$ and all finite subgroups of $\mathbb{T}$. Hence, we have a canonical isomorphism

$$
\operatorname{TR}_{q}^{n}(\mathbb{S} ; p) \cong\left[\mathbb{T} / C_{p^{n-1}}+\wedge S^{q}, S^{0}\right]_{\mathbb{T}}
$$

The groups on the right are well-known, at least for small values of $q$, by (2.1.1). We will use the result for $0 \leqslant q \leqslant 2$. Under the isomorphism above, the multiplicative unit corresponds to the map of suspension $\mathbb{T}$-spectra

$$
e: \mathbb{T} / C_{p^{n-1}+} \rightarrow S^{0}
$$


induced from the projection which collapses $\mathbb{T} / C_{p^{n-1}}$ to the non-basepoint in $S^{0}$. Composition with $e$ defines a map

$$
\left[\mathbb{T} / C_{p^{n-1}+} \wedge S^{q}, \mathbb{T} / C_{p^{n-1}+}\right]_{\mathbb{T}} \rightarrow\left[\mathbb{T} / C_{p^{n-1}+} \wedge S^{q}, S^{0}\right]_{\mathbb{T}}
$$

If $0 \leqslant q \leqslant 2$, the group on the left was described, up to 2 -torsion, in 2.2.4 above. Since $d$ is a derivation, $e \delta$ is zero. This is the only extra relation. Hence:

(i) The maps from $\mathbb{T} / C_{p^{n-1}}$ to $S^{0}$ form a free abelian group of rank $n$ generated by $e f^{s} v^{s}$ with $0 \leqslant s<n$.

(ii) The abelian group of maps from $\mathbb{T} / C_{p^{n-1}+} \wedge S^{1}$ to $S^{0}$ is, up to 2-torsion, the sum for $1 \leqslant s<n$, of a copy of $\mathbb{Z} / p^{s} \mathbb{Z}$ generated by $e f^{s} v^{s} \delta$.

(iii) Up to 2 -torsion, every map from $\mathbb{T} / C_{p^{n-1}+} \wedge S^{2}$ to $S^{0}$ is null.

The unit map $\mathbb{S} \rightarrow \tilde{\mathbb{Z}}$ induces an isomorphism of homotopy groups with $\mathbb{Z}_{(p)}$-coefficients in degrees less than $2 p-3$. And the functor $\operatorname{TR}^{n}(-; p)$ preserves connectivity. Thus we have:

PROPOSITION 2.6.1. - The group $\mathrm{TR}_{0}^{n}\left(\mathbb{Z}_{(p)} ; p\right)$ is a free $\mathbb{Z}_{(p)}$-module of rank $n$ generated by $V^{s}(1), 0 \leqslant s<n$. The group $\operatorname{TR}_{1}^{n}\left(\mathbb{Z}_{(p)} ; p\right)$ is a sum for $1 \leqslant s<n$, of a copy of $\mathbb{Z} / p^{s} \mathbb{Z}$ generated by $d V^{s}(1)$. The group $\operatorname{TR}_{2}^{n}\left(\mathbb{Z}_{(p)} ; p\right)$ is zero.

\section{Polynomial extensions}

3.1. In this section we prove Theorem $\mathrm{C}$ of the introduction. We briefly recall the statement. The ring homomorphism given by the inclusion of the constant polynomials,

$$
\pi: A \rightarrow A[x]
$$

induces a map of Witt complexes over $A$,

$$
f: \mathrm{TR}_{*}(A ; p) \rightarrow \pi_{*} \mathrm{TR}_{*}(A[x] ; p),
$$

where on the right $\pi_{*}$ is the direct image functor. And as part of the structure of a Witt complex, we have the map of pro-rings

$$
\lambda: W .(A[x]) \rightarrow \mathrm{TR}_{0}(A[x] ; p) .
$$

We wish to show that for all $n \geqslant 1$ and $q \geqslant 0$, every element of $\operatorname{TR}_{q}^{n}(A[x] ; p)$ can be written uniquely as a finite sum

$$
\begin{aligned}
& f\left(a_{0,0}^{(n)}\right)+\sum_{j \in \mathbb{N}}\left(f\left(a_{0, j}^{(n)}\right) \lambda\left([x]_{n}^{j}\right)+f\left(b_{0, j}^{(n)}\right) \lambda\left([x]_{n}^{j-1}\right) d \lambda\left([x]_{n}\right)\right) \\
& \quad+\sum_{s=1}^{n-1} \sum_{j \in I_{p}}\left(V^{s}\left(f\left(a_{s, j}^{(n-s)}\right) \lambda\left([x]_{n-s}^{j}\right)\right)+d V^{s}\left(f\left(b_{s, j}^{(n-s)}\right) \lambda\left([x]_{n-s}^{j}\right)\right)\right)
\end{aligned}
$$

with $a_{s, j}^{m} \in \mathrm{TR}_{q}^{m}(A ; p)$ and $b_{s, j}^{(m)} \in \mathrm{TR}_{q-1}^{m}(A ; p)$.

We recall that, by definition, the group $\operatorname{TR}_{q}^{n}(A[x] ; p)$ is the $q$ th homotopy group of the $\mathbb{T}$-spectrum

$$
\rho_{p^{n-1}}^{*} T(A[x])^{C_{p^{n-1}}} .
$$

$4^{\mathrm{e}}$ SÉRIE - TOME $37-2004-\mathrm{N}^{\circ} 1$ 
Let $\Pi=\left\{0,1, x, x^{2}, \ldots\right\}$ be the sub-pointed monoid of $A[x]$ generated by the variable $x$. The $\mathbb{T}$-space $N^{\text {cy }}(\Pi)$ decomposes as a wedge sum

$$
\bigvee_{i \in \mathbb{N}_{0}} N^{\mathrm{cy}}(\Pi, i) \stackrel{\sim}{\rightarrow} N^{\mathrm{cy}}(\Pi)
$$

where the $i$ th summand is the realization of the pointed cyclic subset of $N^{\mathrm{cy}}(\Pi)$ generated by the 0 -simplex 1 , if $i=0$, and by the $(i-1)$-simplex $x \wedge \cdots \wedge x$, if $i>0$. Hence, the $\mathbb{T}$-spectrum (3.1.2) can then be expressed as a wedge sum

$$
\begin{aligned}
& \bigvee_{j \in \mathbb{N}_{0}} \rho_{p^{n-1}}^{*} T(A)^{C_{p^{n-1}}} \wedge N^{\mathrm{cy}}(\Pi, j) \\
& \vee \bigvee_{s=1}^{n-1} \bigvee_{j \in I_{p}} \rho_{p^{s}}^{*}\left(\rho_{p^{n-1-s}}^{*} T(A)^{C_{p^{n-1-s}}} \wedge N^{\mathrm{cy}}(\Pi, j)\right)^{C_{p^{s}}}
\end{aligned}
$$

We recall below how this equivalence is defined and show that the homotopy groups of (3.1.3) are given by the finite sums of the form (3.1.1). This will prove Theorem C.

3.2. We prove in $[10$, Theorem 7.1$]$ that the composite

$$
T(A) \wedge N^{\mathrm{cy}}(\Pi) \stackrel{f \wedge \iota}{\longrightarrow} T(A[x]) \wedge N^{\mathrm{cy}}(A[x]) \stackrel{\mu}{\longrightarrow} T(A[x]),
$$

where $\iota$ is the inclusion, is a natural equivalence of $\mathbb{T}$-spectra. Since $A$ and $\Pi$ are commutative, this equivalence is multiplicative with the componentwise multiplication on the left. This induces an equivalence of $\mathbb{T}$-spectra

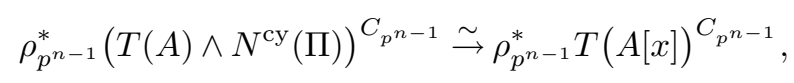

and the wedge decomposition of the $\mathbb{T}$-space $N^{\text {cy }}(\Pi)$ induces one of $\mathbb{T}$-spectra

$$
\bigvee_{i \in \mathbb{N}_{0}} \rho_{p^{n-1}}^{*}\left(T(A) \wedge N^{\mathrm{cy}}(\Pi, i)\right)^{C_{p^{n-1}}} \stackrel{\sim}{\rightarrow} \rho_{p^{n-1}}^{*}\left(T(A) \wedge N^{\mathrm{cy}}(\Pi)\right)^{C_{p^{n-1}}} .
$$

Regrouping the wedge summands after the $p$-adic valuation of the index, we can write the left hand side in the following way.

$$
\begin{aligned}
& \bigvee_{j \in \mathbb{N}_{0}} \rho_{p^{n-1}}^{*}\left(T(A) \wedge N^{\mathrm{cy}}\left(\Pi, p^{n-1} j\right)\right)^{C_{p^{n-1}}} \\
& \vee \bigvee_{s=1}^{n-1} \bigvee_{j \in I_{p}} \rho_{p^{s}}^{*}\left(\rho_{p^{n-1-s}}^{*}\left(T(A)^{C_{p^{n-1-s}}} \wedge N^{\mathrm{cy}}\left(\Pi, p^{n-1-s} j\right)\right)^{C_{p^{n-1-s}}}\right)^{C_{p^{s}}} .
\end{aligned}
$$

Finally, we have the equivalence of $\mathbb{T}$-spectra given by the pairing

$$
\rho_{p^{v}}^{*} T(A)^{C_{p^{v}}} \wedge \rho_{p^{v}}^{*} N^{\mathrm{cy}}\left(\Pi, p^{v} j\right)^{C_{p^{v}}} \stackrel{\sim}{\rightarrow} \rho_{p^{v}}^{*}\left(T(A) \wedge N^{\mathrm{cy}}\left(\Pi, p^{v} j\right)\right)^{C_{p^{v}}}
$$

and the $\mathbb{T}$-equivariant homeomorphism

$$
\Delta: N^{\mathrm{cy}}(\Pi, j) \stackrel{\sim}{\rightarrow} \rho_{p^{v}}^{*} N^{\mathrm{cy}}\left(\Pi, p^{v} j\right)^{C_{p^{v}}} .
$$


This gives the desired equivalence of $\mathbb{T}$-spectra from the wedge sum (3.1.3) to the $\mathbb{T}$-spectrum (3.1.2).

3.3. We first consider the restriction of the equivalence described above to the top summand in (3.1.3). This amounts to a map of $\mathbb{T}$-spectra

$$
\rho_{p^{n-1}}^{*} T(A)^{C_{p^{n-1}}} \wedge N^{\mathrm{cy}}(\Pi) \rightarrow \rho_{p^{n-1}}^{*} T(A[x])^{C_{p^{n-1}}},
$$

which is multiplicative, if the left hand side is given the componentwise multiplication. Hence, the induced map on homotopy groups

$$
\pi_{*}\left(\rho_{p^{n-1}}^{*} T(A)^{C_{p^{n-1}}} \wedge N^{\mathrm{cy}}(\Pi)\right) \rightarrow \mathrm{TR}_{*}^{n}(A[x] ; p)
$$

identifies the left hand side with a sub-differential graded ring of the differential graded ring on the right.

We recall the structure of the $\mathbb{T}$-spaces $N^{\text {cy }}(\Pi, i)$, but see also [10, Section 7.2] and [8, Section 2.2]. The space $N^{\mathrm{cy}}(\Pi, 0)$ is the discrete space $\{0,1\}$, and for $i>0$, there is a canonical $\mathbb{T}$-equivariant homeomorphism $\Lambda^{i-1} / C_{i} \stackrel{\sim}{\rightarrow} N^{\mathrm{cy}}(\Pi, i)$. Here $\Lambda^{i-1}$ is the cyclic standard $(i-1)$-simplex, and the cyclic group $C_{i}$ acts through the (co)cyclic operator $\tau_{i-1}$. We show in [10, Section 7.2] that there is a $\mathbb{T}$-equivariant homeomorphism $\Lambda^{i-1} \approx \mathbb{T} \times \Delta^{i-1}$ such that, on the right, the cocyclic operator acts on $\mathbb{T}$ by multiplication by $e^{2 \pi \sqrt{-1} / i}$ and on $\Delta^{i-1}$ by the affine map which cyclically permutes the vertices. It follows that the inclusion of the barycenter of $\Delta^{i-1}$ gives rise to a strong deformation retract of $\mathbb{T}$-spaces

$$
\mathbb{T} / C_{i+} \stackrel{\sim}{\rightarrow} N^{\mathrm{cy}}(\Pi, i)
$$

The multiplication on $N^{\mathrm{cy}}(\Pi)$ restricts to a pairing of the $i$ th and $i^{\prime}$ th summands to the $\left(i+i^{\prime}\right)$ th summand. The equivalences above are compatible with this pairing in that there is a homotopy commutative diagram of pointed $\mathbb{T}$-spaces

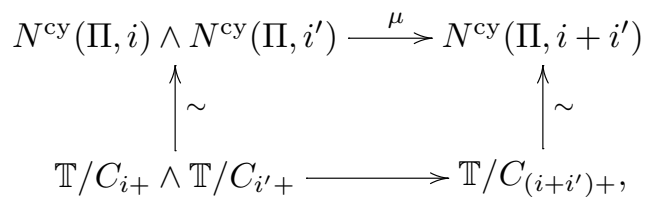

where the lower horizontal map takes $\left(z C_{i}, z^{\prime} C_{i^{\prime}}\right)$ to $\left(z^{i} z^{\prime i^{\prime}}\right)^{1 /\left(i+i^{\prime}\right)} C_{i+i^{\prime}}$.

LEMMA 3.3.1. - The map of differential graded rings

$$
\mathrm{TR}_{*}^{n}(A ; p) \otimes \Omega_{\mathbb{Z}[x]}^{*} \rightarrow \mathrm{TR}_{*}^{n}(A[x] ; p)
$$

which takes $a \otimes 1$ to $f(a)$ and $1 \otimes x$ to $\lambda\left([x]_{n}\right)$ is an isomorphism onto the sub-differential graded ring $\pi_{*}\left(\rho_{p^{n-1}}^{*} T(A)^{C_{p^{n-1}}} \wedge N^{\mathrm{cy}}(\Pi)\right)$.

Proof. - We first show that the map of the statement lands in the indicated sub-differential graded ring. The map of components induced from the composite

$$
\Pi \rightarrow N^{\mathrm{cy}}(\Pi) \stackrel{\Delta}{\rightarrow} \rho_{p^{n-1}} N^{\mathrm{cy}}(\Pi)^{C_{p^{n-1}}} \rightarrow \rho_{p^{n-1}}^{*} T(A[x])^{C_{p^{n-1}}}
$$


takes $x^{i}$ to $\lambda\left([x]_{n}^{i}\right)$. For by definition, the map $A[x] \rightarrow \mathrm{TR}_{0}^{n}(A[x] ; p)$, which takes $g$ to $\lambda\left([g]_{n}\right)$, is the map of components induced by the composite

$$
A[x] \rightarrow N^{\mathrm{cy}}(A[x]) \stackrel{\Delta}{\rightarrow} \rho_{p^{n-1}} N^{\mathrm{cy}}(A[x])^{C_{p^{n-1}}} \rightarrow \rho_{p^{n-1}}^{*} T(A[x])^{C_{p^{n-1}}} .
$$

And the composite

$$
\rho_{p^{n-1}}^{*} T(A)^{C_{p^{n-1}}} \stackrel{\sim}{\rightarrow} \rho_{p^{n-1}}^{*} T(A)^{C_{p^{n-1}}} \wedge N^{\mathrm{cy}}(\Pi, 0) \rightarrow \rho_{p^{n-1}}^{*} T(A[x])^{C_{p^{n-1}}},
$$

where the left hand map is the canonical isomorphism, is equal to the map induced from $\pi: A \rightarrow A[x]$.

Let $x_{i} \in H_{0}\left(N^{\mathrm{cy}}(\Pi)\right.$ be the image of the generator of $\tilde{H}_{0}\left(\mathbb{T} / C_{i+}\right)$ given by the point $C_{i}$. We show that the map of differential graded rings

$$
\Omega_{\mathbb{Z}[x]}^{*} \stackrel{\sim}{\rightarrow} \tilde{H}_{*}\left(N^{c y}(\Pi)\right),
$$

which takes $x$ to $x_{1}$ is an isomorphism. The map in homology induced by the product

$$
\mathbb{T} / C_{i+} \wedge \mathbb{T} / C_{i^{\prime}+} \rightarrow \mathbb{T} / C_{\left(i+i^{\prime}\right)+}
$$

takes the cycles $C_{i} \otimes C_{i^{\prime}}$ to the cycle $C_{i+i^{\prime}}$, and hence, $x_{i} x_{i^{\prime}}=x_{i+i^{\prime}}$. This proves that the map is an isomorphism in degree zero. To prove that it is an isomorphism in degree one, it suffices to show that $x^{i-1} d x$ is a generator of $\tilde{H}_{1}\left(N^{\mathrm{cy}}(\Pi, i)\right)$. But $i x^{i-1} d x=d\left(x^{i}\right)$ and $d\left(x^{i}\right)$ is $i$ times a generator; compare $[9,(2.1 .2)]$ and (2.2.1) and (2.2.2) above.

Since the homology of $N^{\mathrm{cy}}(\Pi)$ is torsion free, the spectral sequence obtained from the skeleton filtration of $N^{\mathrm{cy}}(\Pi)$ takes the form

$$
E^{2}=\mathrm{TR}_{*}^{n}(A ; p) \otimes \tilde{H}_{*}\left(N^{\mathrm{cy}}(\Pi)\right) \Rightarrow \pi_{*}\left(\rho_{p^{n-1}}^{*} T(A)^{C_{p^{n-1}}} \wedge N^{\mathrm{cy}}(\Pi)\right) .
$$

The spectral sequence is concentrated on the lines $E_{0, *}^{2}$ and $E_{1, *}^{2}$, and hence all differentials are zero. In particular, the edge homomorphism is an isomorphism. We can write this as the composite

$$
\mathrm{TR}_{*}^{n}(A ; p) \otimes \mathbb{Z}[x] \rightarrow \mathrm{TR}_{*}^{n}(A ; p) \otimes \Omega_{\mathbb{Z}[x]}^{*} \rightarrow \pi_{*}\left(\rho_{p^{n-1}}^{*} T(A)^{C_{p^{n-1}}} \wedge N^{\mathrm{cy}}(\Pi)\right),
$$

where the left hand map is the inclusion and the right hand map is the map of the statement. It remains to show that the induced map

$$
\left(\mathrm{TR}_{*}^{n}(A ; p) \otimes \Omega_{\mathbb{Z}[x]}^{*}\right) /\left(\mathrm{TR}_{*}^{n}(A ; p) \otimes \mathbb{Z}[x]\right) \rightarrow \mathrm{TR}_{*}(A ; p) \otimes \tilde{H}_{1}\left(N^{\mathrm{cy}}(\Pi)\right)
$$

is an isomorphism. The domain and range are both free $\operatorname{TR}_{*}(A ; p) \otimes \mathbb{Z}[x]$-modules of rank one. And the generator $1 \otimes d x=d(1 \otimes x)$ on the left maps to the generator $1 \otimes d x$ on the right. This completes the proof.

3.4. It remains to prove that the homotopy groups of the lower wedge summands in (3.1.3) correspond to the lower summands in (3.1.1). This follows from Lemma 3.3.1 and the following

Lemma 3.4.1. - Let $T$ be a $\mathbb{T}$-spectrum, let $j \in I_{p}$, and let $\iota: C_{j} / C_{j} \rightarrow \mathbb{T} / C_{j}$ be the canonical inclusion. Then for all integers $q$ and $v \geqslant 0$, the map

$$
V^{v} \iota+d V^{v} \iota: \pi_{q}(T) \oplus \pi_{q-1}(T) \stackrel{\sim}{\rightarrow} \pi_{q}\left(T \wedge \mathbb{T} / C_{j+}\right)^{C_{p} v}
$$


is an isomorphism.

Proof. - If $X$ is a pointed $C_{p^{v}}$-CW-complex, the skeleton filtration gives rise to a spectral sequence

$$
E_{s, t}^{1}=\pi_{s+t}\left(\left(T \wedge X_{s} / X_{s-1}\right)^{C_{p^{v}}}\right) \Rightarrow \pi_{s+t}\left((T \wedge X)^{C_{p^{v}}}\right) .
$$

And if the $C_{p^{v}}$-action on $X$ is free away from the base point, the canonical map

$$
\pi_{s+t}\left((T \wedge X)^{C_{p^{v}}}\right) \stackrel{\sim}{\rightarrow}\left(\pi_{s+t}(T \wedge X)\right)^{C_{p} v}
$$

is an isomorphism. And since, non-equivariantly, $X_{s} / X_{s-1}$ is a wedge of $s$-spheres, there are $C_{p^{v}}$-equivariant isomorphisms

$$
\pi_{s+t}\left(T \wedge X_{s} / X_{s-1}\right) \stackrel{\sim}{\leftarrow} \pi_{t}(T) \otimes \pi_{s}\left(X_{s} / X_{s-1}\right) \stackrel{\sim}{\rightarrow} \pi_{t}(T) \otimes \tilde{H}_{s}\left(X_{s} / X_{s-1}\right) .
$$

Here the left hand map is the natural pairing and the right hand map is the Hurewitz homomorphism. Hence, we have a natural isomorphism of chain complexes

$$
E_{*, t}^{1} \cong\left(\pi_{t}(T) \otimes \tilde{C}_{*}(X)\right)^{C_{p^{v}}}
$$

where $\tilde{C}_{*}(X)$ is the reduced cellular complex of $X$.

In the case at hand, we give $X=\mathbb{T} / C_{j}$ a $C_{p^{v}}$-CW-structure with one free cell in dimensions zero and one. Let $g$ be the generator $e^{2 \pi i / p^{v}} \in C_{p^{v}}$. Then the attaching maps

$$
\alpha_{s}: D^{s} \times C_{p^{v}} \rightarrow X_{s}
$$

are given by $\alpha_{0}\left(g^{n}\right)=g^{n} C_{j}$ and $\alpha_{1}\left(x, g^{n}\right)=g^{n} e^{\pi i(x+1) / p^{v}} C_{j}$, respectively. We define $W(j)$ to be the complex of $\mathbb{Z}\left[C_{p^{v}}\right]$-modules which in degrees $s=0,1$ is a free $\mathbb{Z}\left[C_{p^{v}}\right]$-module on a single generator $y_{s}$ with differential $d\left(y_{1}\right)=\left(g^{j}-1\right) y_{0}$. Then the attaching maps define an isomorphism of complexes

$$
W(j) \stackrel{\sim}{\rightarrow} C_{*}\left(\mathbb{T} / C_{j}\right),
$$

which takes $y_{s}$ to the image of the generator of $H_{s}\left(D^{s}, \partial D^{s}\right)$ corresponding to the standard orientation of $D^{s}$. Since $T$ is a $\mathbb{T}$-spectrum the action of $C_{p^{v}}$ on $\pi_{t}(T)$ is trivial. Hence

$$
E_{s, t}^{1} \cong \pi_{t}(T) \cdot N y_{s}, \quad s=0,1
$$

where $N \in \mathbb{Z}\left[C_{p^{v}}\right]$ is the norm element. Moreover,

$$
\left(g^{j}-1\right) N=N-N=0,
$$

so the $d^{1}$-differential vanishes. The higher differentials are zero for degree reasons, and hence, the groups $\pi_{q}\left(\left(T \wedge \mathbb{T} / C_{j+}\right)^{C_{p^{v}}}\right)$ are as stated, at least up to an extension.

It remains to show that the map of the statement is an isomorphism. We also have a spectral sequence

$$
E_{s, t}^{1}=\pi_{s+t}\left(T \wedge X_{s} / X_{s-1}\right) \Rightarrow \pi_{s+t}(T \wedge X) .
$$

In the case at hand, the same reasoning as above gives a natural isomorphism of complexes

$$
E_{*, t}^{1} \cong \pi_{t}(T) \otimes W(j)
$$


It follows that $E_{0, t}^{2} \cong \pi_{t}(T) \cdot y_{0}$ and $E_{1, t}^{2} \cong \pi_{t}(T) \cdot N y_{1}$. The map

$$
V^{v}: \pi_{*}\left(T \wedge \mathbb{T} / C_{j+}\right) \rightarrow \pi_{*}\left(\left(T \wedge \mathbb{T} / C_{j+}\right)^{C_{p^{v}}}\right)
$$

induces a map of spectral sequences. With our identification of the $E^{1}$-terms, this corresponds to the norm map

$$
N: \pi_{t}(T) \otimes W(j) \rightarrow\left(\pi_{t}(T) \otimes W(j)\right)^{C_{p^{v}}} .
$$

The induced map on $E_{0, t}^{2}$ maps $x \cdot y_{0}$ to $x \cdot N y_{0}$, and hence, is an isomorphism. We also note that the induced map on $E_{1, t}^{2}$ maps $x \cdot N y_{1}$ to $x \cdot N N y_{1}=p^{v} x \cdot N y_{1}$.

Finally, we show that under the above identifications, the composite

$$
E_{0, t}^{2} \longmapsto \pi_{t}\left(\left(T \wedge \mathbb{T} / C_{j+}\right)^{C_{p^{v}}}\right) \stackrel{d}{\rightarrow} \pi_{t+1}\left(\left(T \wedge \mathbb{T} / C_{j+}\right)^{C_{p^{v}}}\right) \rightarrow E_{1, t}^{2}
$$

takes $x \cdot N y_{0}$ to $j x \cdot N y_{1}$. By naturality, we may assume that $\pi_{t}(T)$ is torsion free. For given $x \in \pi_{t}(T)$, we can find a map of $\mathbb{T}$-spectra $S^{t} \wedge \mathbb{T}_{+} \rightarrow T$ such that the induced map on homotopy groups maps a generator of $\pi_{t}\left(S^{t} \wedge \mathbb{T}_{+}\right) \cong \mathbb{Z}$ to $x$. Hence, it suffices to show that the composite

$$
E_{0, t}^{2} \longmapsto \pi_{t}\left(\left(T \wedge \mathbb{T} / C_{j+}\right)^{C_{p^{v}}}\right) \stackrel{p^{v} d}{\longrightarrow} \pi_{t+1}\left(\left(T \wedge \mathbb{T} / C_{j+}\right)^{C_{p^{v}}}\right) \rightarrow E_{1, t}^{2}
$$

takes $x \cdot N y_{0}$ to $p^{v} j x \cdot N y_{1}$. But $x \cdot N y_{0}=V^{v}\left(x \cdot y_{0}\right)$, and hence it suffices to show that the composite

$$
E_{0, t}^{2} \longmapsto \pi_{t}\left(T \wedge \mathbb{T} / C_{j+}\right) \stackrel{d}{\rightarrow} \pi_{t+1}\left(T \wedge \mathbb{T} / C_{j+}\right) \rightarrow E_{1, t}^{2}
$$

takes $x \cdot y_{0}$ to $j x \cdot N y_{1}$. This is the statement that the map

$$
H_{1}(\mathbb{T}) \otimes H_{0}\left(\mathbb{T} / C_{j}\right) \stackrel{\times}{\rightarrow} H_{1}\left(\mathbb{T} \times \mathbb{T} / C_{j}\right) \stackrel{\mu}{\rightarrow} H_{1}\left(\mathbb{T} / C_{j}\right)
$$

takes $[\mathbb{T}] \otimes y_{0}$ to $j N y_{1}$, which is standard.

\section{The functor $P(-)$}

4.1. We first evaluate the Witt ring $W_{n}(A[x])$.

Lemma 4.1.1. - Let $A$ be a ring. Then every element $f^{(n)} \in W_{n}(A[x])$ may be written uniquely

$$
f^{(n)}=\sum_{j \in \mathbb{N}_{0}} a_{0, j}^{(n)}[x]_{n}^{j}+\sum_{s=1}^{n-1} \sum_{j \in I_{p}} V^{s}\left(a_{s, j}^{(n-s)}[x]_{n-s}^{j}\right)
$$

with $a_{s, j}^{(n-s)} \in W_{n-s}(A)$, and where all but finitely many $a_{s, j}^{(n-s)}$ are zero.

Proof. - Let $Q_{n}$ be the set of expressions of the form

$$
f^{(n)}=\sum_{j \in \mathbb{N}_{0}} a_{0, j}^{(n)}[x]_{n}^{j}+\sum_{s=1}^{n-1} \sum_{j \in I_{p}} V^{s}\left(a_{s, j}^{(n-s)}[x]_{n-s}^{j}\right),
$$


with the components $a_{s, j}^{(n-s)} \in W_{n-s}(A)$, all but finitely many of which are zero. We consider $Q_{n}$ an abelian group under componentwise addition. Moreover, interpreting the expression $f^{(n)}$ as an element of $W_{n}(A[x])$ defines an additive map

$$
Q_{n} \rightarrow W_{n}(A[x]),
$$

and it is clear that this is an isomorphism, for $n=1$. The proof of the general case is by induction on $n$ based on the diagram

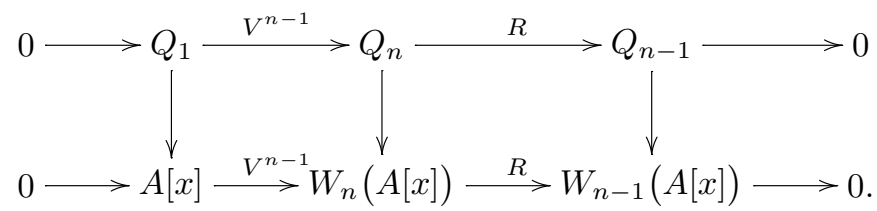

The lower sequence is exact and the right and left vertical maps are isomorphisms by the inductive hypothesis. It thus suffices to show that the upper sequence is exact. The restriction $R: Q_{n} \rightarrow Q_{n-1}$

$$
R f^{(n)}=\sum_{j \in \mathbb{N}_{0}} R a_{0, j}^{(n)}[x]_{n-1}^{j}+\sum_{s=1}^{n-2} \sum_{j \in I_{p}} V^{s}\left(R a_{s, j}^{(n-s)}[x]_{n-1-s}\right),
$$

is surjective since $R: W_{n}(A) \rightarrow W_{n-1}(A)$ is surjective, and $V^{n-1}: Q_{1} \rightarrow Q_{n}$,

$$
V^{n-1}\left(f^{(1)}\right)=\sum_{j \in \mathbb{N}_{0}} V^{n-1}\left(a_{0, p^{n-1} j}^{(1)}\right)[x]_{n}^{j}+\sum_{s=1}^{n-1} \sum_{j \in I_{p}} V^{s}\left(V^{n-1-s}\left(a_{0, p^{n-1-s} j}^{(1)}\right)[x]_{n-s}^{j}\right),
$$

is injective since the maps $V^{m}: A \rightarrow W_{m}(A), 1 \leqslant m<n$, are injective. It is also clear that the composite $R V^{n-1}$ is zero. Finally, $R f^{(n)}$ vanishes if and only if each $a_{s, i}^{(n-s)}$ is in the kernel of $R: W_{n-s}(A) \rightarrow W_{n-1-s}(A)$, or equivalently, if $a_{s, j}^{(n)}=V^{n-1-s}\left(a_{p^{n-1-s} j}^{(1)}\right)$. Hence $f^{(n-1)}=V^{n-1}\left(\sum_{j \in \mathbb{N}_{0}} a_{j}^{(1)} x^{j}\right)$.

4.2. Let $\pi: A \rightarrow A[x]$ be the inclusion of the constant polynomials. In this paragraph, we give an explicit construction of the inverse image functor

$$
\pi^{*}: \mathcal{W}_{A} \rightarrow \mathcal{W}_{A[x]}
$$

Let $E=E^{*}$. be a Witt complex over $A$, we let $P(E)=P(E)^{*}$ be the pro-graded abelian group with $P(E)_{n}^{q}$ equal to the set of all (finite) formal sums of the form

$$
\begin{aligned}
& \sum_{j \in \mathbb{N}_{0}} a_{0, j}^{(n)}[x]_{n}^{j}+\sum_{j \in \mathbb{N}} b_{0, j}^{(n)}[x]_{n}^{j-1} d[x]_{n} \\
& \sum_{s=1}^{n-1} \sum_{j \in I_{p}}\left(V^{s}\left(a_{s, j}^{(n-s)}[x]_{n-s}^{j}\right)+d V^{s}\left(b_{s, j}^{(n-s)}[x]_{n-s}^{j}\right)\right),
\end{aligned}
$$

with components $a_{s, j}^{(m)} \in E_{m}^{q}$ and $b_{s, j}^{(m)} \in E_{m}^{q-1}$. Addition is component-wise, and the structure maps in the pro-system are induced from the ones in $E$. Given a Witt complex $E^{\prime}=E^{\prime *}$ over 
$A[x]$ and a map $f: E \rightarrow \pi_{*} E^{\prime}$ of Witt complexes over $A$, the induced strict map of pro-graded abelian groups

$$
\tilde{f}: P(E) \rightarrow E^{\prime}
$$

maps the formal sum (4.2.1) to the sum

$$
\begin{aligned}
& \sum_{j \in \mathbb{N}_{0}} f\left(a_{0, j}^{(n)}\right) \lambda^{\prime}\left([x]_{n}^{j}\right)+\sum_{j \in \mathbb{N}} f\left(b_{0, j}^{(n)}\right) \lambda^{\prime}\left([x]_{n}^{j-1}\right) d \lambda^{\prime}\left([x]_{n}\right) \\
& \sum_{s=1}^{n-1} \sum_{j \in I_{p}}\left(V^{s}\left(f\left(a_{s, j}^{(n-s)}\right) \lambda^{\prime}\left([x]_{n-s}^{j}\right)\right)+d V^{s}\left(f\left(b_{s, j}^{(n-s)}\right) \lambda^{\prime}\left([x]_{n-s}^{j}\right)\right)\right)
\end{aligned}
$$

in $E_{q}^{\prime n}$. The requirement that for all $E^{\prime}$ in $\mathcal{W}_{A^{\prime}}$, this be a map of Witt complexes leaves only one possible way to define a product, a differential, and the maps $F$ and $V$ on $P(E)$. We give the formulas which define these operations. There are several special cases to consider, and to enhance readability, we suppress all non-essential indices. It is understood that the formulas are valid for all possible values of non-restricted indices.

The differential

$$
d: P(E)_{n}^{q} \rightarrow P(E)_{n}^{q+1}
$$

is given by the following formulas:

$$
\begin{aligned}
d\left(V^{s}\left(a[x]^{j}\right)\right) & =(d a)[x]^{j}+(-1)^{q} j a[x]^{j-1} d[x], \quad \text { if } s=0, \\
& =d V^{s}\left(a[x]^{j}\right), \quad \text { if } s>0 ; \\
d\left(b[x]^{j-1} d[x]\right) & =(d b)[x]^{j-1} d[x] ; \\
d\left(d V^{s}\left(b[x]^{j}\right)\right) & =0 .
\end{aligned}
$$

The Frobenius

$$
F: P(E)_{n}^{q} \rightarrow P(E)_{n-1}^{q}
$$

is given by

$$
\begin{aligned}
F\left(V^{s}\left(a[x]^{j}\right)\right) & =F(a)[x]^{p j}, \quad \text { if } s=0, \\
& =p V^{s-1}\left(a[x]^{j}\right), \quad \text { if } s>0 ; \\
F\left(b[x]^{j-1} d[x]\right) & =F(b)[x]^{p j-1} d[x] ; \\
F\left(d V^{s}\left(b[x]^{j}\right)\right) & =d V^{s-1}\left(b[x]^{j}\right) .
\end{aligned}
$$

The Verschiebung

$$
V: P(E)_{n-1}^{q} \rightarrow P(E)_{n}^{q}
$$

is given by

$$
\begin{aligned}
V\left(V^{s}\left(a[x]^{j}\right)\right) & =V^{s+1}\left(a[x]^{j}\right), \\
V\left(b[x]^{j-1} d[x]\right) & =(-1)^{q-1} \frac{p}{j} d V\left(b[x]^{j}\right)-(-1)^{q-1} \frac{1}{j} V\left((d b)[x]^{j}\right), \quad \text { if } v_{p}(j)=0, \\
& =V(b)[x]^{p^{-1} j-1} d[x], \quad \text { if } v_{p}(j)>0 ; \\
V\left(d V^{s}\left(b[x]^{j}\right)\right) & =p d V^{s+1}\left(b[x]^{j}\right) .
\end{aligned}
$$


The product

$$
\mu_{n}: P(E)_{n}^{q} \otimes P(E)_{n}^{q^{\prime}} \rightarrow P(E)_{n}^{q+q^{\prime}}
$$

\section{is given by}

$$
\begin{aligned}
& V^{s}\left(a[x]^{j}\right) V^{s^{\prime}}\left(a^{\prime}[x]^{j^{\prime}}\right) \\
& =p^{s} V^{s^{\prime}}\left(F^{s^{\prime}-s}(a) a^{\prime}[x]^{p^{s^{\prime}-s} j+j^{\prime}}\right), \quad \text { if } 0 \leqslant s<s^{\prime}, \\
& =p^{s} V^{s^{\prime}-v}\left(V^{v}\left(a a^{\prime}\right)[x]^{p^{-v}\left(j+j^{\prime}\right)}\right), \quad \text { if } 0 \leqslant s=s^{\prime} \text { and } v=v_{p}\left(j+j^{\prime}\right)<s^{\prime} \text {, } \\
& =p^{s} V^{s^{\prime}}\left(a a^{\prime}\right)[x]^{p^{-s^{\prime}}\left(j+j^{\prime}\right)}, \quad \text { if } 0 \leqslant s=s^{\prime} \text { and } v=v_{p}\left(j+j^{\prime}\right) \geqslant s^{\prime} \text {; } \\
& V^{s}\left(a[x]^{j}\right) b^{\prime}[x]^{j^{\prime}-1} d[x]=a b^{\prime}[x]^{j+j^{\prime}-1} d[x], \quad \text { if } s=0, \\
& =(-1)^{q+q^{\prime}} \frac{1}{j+p^{s} j^{\prime}} V^{s}\left(d\left(a F^{s}\left(b^{\prime}\right)\right)[x]^{j+p^{s} j^{\prime}}\right) \\
& -(-1)^{q+q^{\prime}} \frac{p^{s}}{j+p^{s} j^{\prime}} d V^{s}\left(a F^{s}\left(b^{\prime}\right)[x]^{j+p^{s} j^{\prime}}\right), \quad \text { if } 0<s ; \\
& d V^{s}\left(b[x]^{j}\right) V^{s^{\prime}}\left(a^{\prime}[x]^{j^{\prime}}\right) \\
& =V^{s^{\prime}}\left(F^{s^{\prime}-s}(d b) a^{\prime}[x]^{p^{s^{\prime}-s} j+j^{\prime}}\right)+\frac{p^{s^{\prime}} j}{p^{s^{\prime}-s} j+j^{\prime}} d V^{s^{\prime}}\left(F^{s^{\prime}-s}(b) a^{\prime}[x]^{p^{s^{\prime}-s} j+j^{\prime}}\right) \\
& -\frac{j}{p^{s^{\prime}-s} j+j^{\prime}} V^{s^{\prime}}\left(d\left(F^{s^{\prime}-s}(b) a^{\prime}\right)[x]^{p^{s^{\prime}-s} j+j^{\prime}}\right), \quad \text { if } 0 \leqslant s<s^{\prime}, \\
& =V^{s^{\prime}-v}\left(V^{v}\left(d(b) a^{\prime}\right)[x]^{p^{-v}\left(j+j^{\prime}\right)}\right)+\frac{p^{s^{\prime}} j}{j+j^{\prime}} d V^{s^{\prime}-v}\left(V^{v}\left(b a^{\prime}\right)[x]^{p^{-v}\left(j+j^{\prime}\right)}\right) \\
& -\frac{p^{v} j}{j+j^{\prime}} V^{s^{\prime}-v}\left(d V^{v}\left(b a^{\prime}\right)[x]^{p^{-v}\left(j+j^{\prime}\right)}\right), \quad \text { if } 0<s=s^{\prime} \text { and } v=v_{p}\left(j+j^{\prime}\right)<s^{\prime}, \\
& =V^{s^{\prime}}\left(d(b) a^{\prime}\right)[x]^{p^{-s^{\prime}}\left(j+j^{\prime}\right)} \\
& -(-1)^{q+q^{\prime}} j V^{s^{\prime}}\left(b a^{\prime}\right)[x]^{p^{-s^{\prime}}\left(j+j^{\prime}\right)-1} d[x], \quad \text { if } 0<s=s^{\prime} \text { and } v=v_{p}\left(j+j^{\prime}\right) \geqslant s^{\prime}, \\
& =(-1)^{q} V^{s}\left(b F^{s-s^{\prime}}\left(d a^{\prime}\right)[x]^{j+p^{s-s^{\prime}} j^{\prime}}\right)+\frac{p^{s^{\prime}} j}{j+p^{s-s^{\prime} j^{\prime}}} d V^{s}\left(b F^{s-s^{\prime}}\left(a^{\prime}\right)[x]^{j+p^{s-s^{\prime}} j^{\prime}}\right) \\
& +\frac{j^{\prime}}{j+p^{s-s^{\prime}} j^{\prime}} V^{s}\left(d\left(b F^{s-s^{\prime}}\left(a^{\prime}\right)\right)[x]^{j+p^{s-s^{\prime}} j^{\prime}}\right), \quad \text { if } 0 \leqslant s^{\prime}<s ; \\
& b[x]^{j-1} d[x] b^{\prime}[x]^{j^{\prime}-1} d[x]=0 ; \\
& d V^{s}\left(b[x]^{j}\right) b^{\prime}[x]^{j^{\prime}-1} d[x]=(-1)^{q-1+q^{\prime}} \frac{1}{j+p^{s} j^{\prime}} d V^{s}\left(d b F^{s}\left(b^{\prime}\right)[x]^{j+p^{s} j^{\prime}}\right) \\
& +(-1)^{q^{\prime}} \frac{1}{j+p^{s} j^{\prime}} V^{s}\left(d b F^{s}\left(d b^{\prime}\right)[x]^{j+p^{s} j^{\prime}}\right), \quad \text { if } 0<s ; \\
& d V^{s}\left(b[x]^{j}\right) d V^{s^{\prime}}\left(b^{\prime}[x]^{j^{\prime}}\right) \\
& =(-1)^{q} d V^{s^{\prime}}\left(F^{s^{\prime}-s}(d b) b^{\prime}[x]^{s^{s^{\prime}-s} j+j^{\prime}}\right) \\
& -(-1)^{q} \frac{j}{p^{s^{\prime}-s} j+j^{\prime}} d V^{s^{\prime}}\left(d\left(F^{s^{\prime}-s}(b) b^{\prime}\right)[x]^{p^{s^{\prime}-s} j+j^{\prime}}\right), \quad \text { if } 0<s<s^{\prime} .
\end{aligned}
$$

Finally, the map 


$$
\lambda: W_{n}(A[x]) \rightarrow P(E)_{n}^{0}
$$

is given by

$$
\lambda\left(V^{s}\left(a[x]^{j}\right)\right)=V^{s}\left(\lambda(a)[x]^{j}\right) .
$$

Here we use Lemma 4.1.1 to write every element of $W_{n}(A[x])$ as a sum of elements of the form $V^{s}\left(a[x]^{j}\right)$ with $a \in W_{n-s}(A)$.

THEOREM 4.2.8. - The formulas (4.2.3)-(4.2.7) make $P\left(W . \Omega_{A}^{*}\right)$ a Witt complex over $A[x]$. Moreover, the canonical map

$$
W . \Omega_{A[x]}^{*} \rightarrow P\left(W . \Omega_{A}^{*}\right)
$$

is an isomorphism.

Proof. - Suppose that $P\left(W . \Omega_{A}^{*}\right)$ is a Witt complex over $A[x]$. Then the composition of the map of the statement and the map

$$
P\left(W . \Omega_{A}^{*}\right) \rightarrow W . \Omega_{A[x]}^{*}
$$

induced from the unique map $W \cdot \Omega_{A}^{*} \rightarrow \pi_{*} W \cdot \Omega_{A[x]}^{*}$ is a self map of $W \cdot \Omega_{A[x]}^{*}$. But the only self map of an initial object is the identity, and hence the map of the statement is injective. It is surjective because the composition

$$
\Omega_{W \cdot(A[x])}^{*} \rightarrow W \cdot \Omega_{A[x]}^{*} \rightarrow P\left(W \cdot \Omega_{A}^{*}\right)
$$

is surjective. We proceed to prove that $P\left(W . \Omega_{A}^{*}\right)$ is a Witt complex. The proof is in two steps.

Suppose first that $A$ is a finitely generated polynomial algebra over $\mathbb{Z}_{(p)}$. We prove by induction on the number of variables that $P\left(W . \Omega_{A}^{*}\right)$ is a Witt complex and that the canonical map

$$
W . \Omega_{A[x]}^{*} \rightarrow \mathrm{TR}_{*}(A[x] ; p)
$$

is injective. The proof of the basic case $A=\mathbb{Z}_{(p)}$ and the induction step are similar. In both cases, the starting point is the fact that the canonical map

$$
W . \Omega_{A}^{*} \rightarrow \mathrm{TR}_{*}^{*}(A ; p)
$$

is injective. We proved in Example 1.2.4. and Proposition 2.6.1 that this is true if $A=\mathbb{Z}_{(p)}$, and in the induction step, it follows from the previous case. It follows that the induced map

$$
P\left(W . \Omega_{A}^{*}\right) \rightarrow P\left(\mathrm{TR}_{*}(A ; p)\right)
$$

is injective. But the canonical map

$$
P\left(\mathrm{TR}_{*}(A ; p)\right) \rightarrow \mathrm{TR}_{*}(A[x] ; p)
$$

is an isomorphism by Theorem $\mathrm{C}$, and hence the canonical map

$$
P\left(W . \Omega_{A}^{*}\right) \rightarrow \mathrm{TR}_{*}(A[x] ; p)
$$

is injective. The definitions (4.2.3)-(4.2.7) were made such that this map is multiplicative and commutes with the maps $d, F, V$, and $\lambda$. Hence, since $\operatorname{TR}_{*}(A[x] ; p)$ is a Witt complex over 
$A[x]$, so is $P\left(W . \Omega_{A}^{*}\right)$. Finally, in the diagram

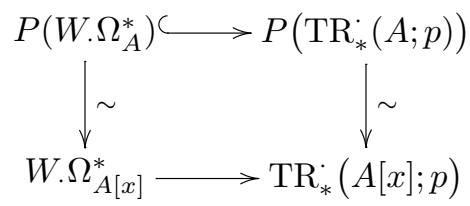

the top horizontal map is injective and the vertical maps are isomorphisms. Hence the lower horizontal map is injective.

Let $A$ be a general $\mathbb{Z}_{(p)}$-algebra. To show that $P\left(W . \Omega_{A}^{*}\right)$ is a Witt complex over $A[x]$ we must verify a number of relations. Each relation involves only a finite number of elements from $W \cdot \Omega_{A}^{*}$. Hence, it suffices to show that given a finite set of elements of $W \cdot \Omega_{A}^{*}$, we can find a ring homomorphism $A^{\prime} \rightarrow A$ from a finitely generated polynomial algebra over $\mathbb{Z}_{(p)}$ such that this finite set of elements is in the image of the induced map

$$
W . \Omega_{A^{\prime}}^{*} \rightarrow W \cdot \Omega_{A}^{*} .
$$

Indeed, we already know that $P\left(W . \Omega_{A^{\prime}}^{*}\right)$ is a Witt complex, so the corresponding relations hold there. It is clear that given a finite set of elements of $\Omega_{W \text {.(A) }}^{*}$, we can find $A^{\prime} \rightarrow A$, where $A^{\prime}$ is a finitely generated $Z_{(p)}$-algebra, such that these elements are in the images of $\Omega_{\left.W \text {.(A } A^{\prime}\right)}^{*} \rightarrow \Omega_{W \text {.(A) }}^{*}$. And since $\Omega_{W \text {.(A) }}^{*} \rightarrow W . \Omega_{A}^{*}$ is surjective, we are done.

Proposition 4.2.9. - Let E be a Witt complex over A. Then the product (4.2.6) and the differential (4.2.3) make $P(E)$ a pro-differential graded ring, and the map (4.2.7) is a map of pro-rings. The Frobenius (4.2.4) is multiplicative, and the Frobenius (4.2.4) and Verschiebung (4.2.5) satisfy Frobenius reciprocity.

Proof. - This is a long straightforward but tedious calculation which we omit. Along the way one uses the relations among $F, d$ and $V$ in $E$. As an example, we verify the associativity relation

$$
\left(d V^{s}\left(a[x]^{j}\right) b^{\prime}[x]^{j^{\prime}-1} d[x]\right) b^{\prime \prime}[x]^{j^{\prime \prime}-1} d[x]=d V^{s}\left(a[x]^{j}\right)\left(b^{\prime}[x]^{j^{\prime}-1} d[x] b^{\prime \prime}[x]^{j^{\prime \prime}-1} d[x]\right) .
$$

The right hand side, by definition, is zero, so we must show that the left hand side, too, is zero. This is easy if $s=0$, so we consider the case $s>0$. The product in the parenthesis is equal to the unit $(-1)^{q^{\prime}} /\left(j+p^{s} j^{\prime}\right)$ times

$$
(-1)^{q-1} d V^{s}\left(d b F^{s}\left(b^{\prime}\right)[x]^{j+p^{s} j^{\prime}}\right)+V^{s}\left(d b F^{s}\left(d b^{\prime}\right)[x]^{j+p^{s} j^{\prime}}\right) .
$$

If we multiply the first summand by $b^{\prime \prime}[x]^{j^{\prime \prime}-1} d[x]$ from the right, we get the unit $(-1)^{q^{\prime \prime}} /\left(j+p^{s} j^{\prime}+p^{s} j^{\prime \prime}\right)$ times

$$
\begin{aligned}
(-1)^{q^{\prime}} & d V^{s}\left(d\left(d b F^{s}\left(b^{\prime}\right)\right) F^{s}\left(b^{\prime \prime}\right)[x]^{j+p^{s} j^{\prime}+p^{s} j^{\prime \prime}}\right) \\
& +(-1)^{q-1} V^{s}\left(d\left(d b F^{s}\left(b^{\prime}\right)\right) F^{s}\left(d b^{\prime \prime}\right)[x]^{j+p^{s} j^{\prime}+p^{s} j^{\prime \prime}}\right) \\
= & (-1)^{q+q^{\prime}} p^{s} d V^{s}\left(d b F^{s}\left(d b^{\prime}\right) F^{s}\left(b^{\prime \prime}\right)[x]^{j+p^{s} j^{\prime}+p^{s} j^{\prime \prime}}\right) \\
& -p^{s} V^{s}\left(d b F^{s}\left(d b^{\prime}\right) F^{s}\left(d b^{\prime \prime}\right)[x]^{j+p^{s} j^{\prime}+p^{s} j^{\prime \prime}}\right) .
\end{aligned}
$$

Here we use the relation $d F^{s}=p^{s} F^{s} d$ in $E$. Similarly, the product of the second summand with $b^{\prime \prime}[x]^{j^{\prime \prime}-1} d[x]$ is the same unit $(-1)^{q^{\prime \prime}} /\left(j+p^{s} j^{\prime}+p^{s} j^{\prime \prime}\right)$ times 


$$
\begin{aligned}
(-1)^{q+q^{\prime}} V^{s}\left(d\left(d b F^{s}\left(d b^{\prime}\right)\right) F^{s}\left(b^{\prime \prime}\right)[x]^{j+p^{s} j^{\prime}+p^{s} j^{\prime \prime}}\right) & \\
& -(-1)^{q+q^{\prime}} p^{s} d V^{s}\left(d b F^{s}\left(d b^{\prime}\right) F^{s}\left(b^{\prime \prime}\right)[x]^{j+p^{s} j^{\prime}+p^{s} j^{\prime \prime}}\right) \\
= & p^{s} V^{s}\left(d b F^{s}\left(d b^{\prime}\right) F^{s}\left(d b^{\prime \prime}\right)[x]^{j+p^{s} j^{\prime}+p^{s} j^{\prime \prime}}\right) \\
& -(-1)^{q+q^{\prime}} p^{s} d V^{s}\left(d b F^{s}\left(d b^{\prime}\right) F^{s}\left(b^{\prime \prime}\right)[x]^{j+p^{s} j^{\prime}+p^{s} j^{\prime \prime}}\right) .
\end{aligned}
$$

The sums cancel as desired.

Proof of Theorem B. - To show that $P(E)$ is a Witt complex over $A[x]$, it remains to verify that for all $f \in A[x]$,

$$
F d \lambda\left([f]_{n}\right)=\lambda\left([f]_{n-1}\right)^{p-1} d \lambda\left([f]_{n-1}\right) .
$$

This is a relation between elements in the image of the map $P\left(W . \Omega_{A}^{*}\right) \rightarrow P(E)$ induced by the unique map $W . \Omega_{A}^{*} \rightarrow E$. And the relation holds in $P\left(W . \Omega_{A}^{*}\right)$ by Theorem 4.2.8. Hence it also holds in $P(E)$.

The second part of the theorem is equivalent to the statement that the map

$$
\operatorname{Hom}_{\mathcal{W}_{A}}\left(E, \pi_{*} E^{\prime}\right) \rightarrow \operatorname{Hom}_{\mathcal{W}_{A[x]}}\left(P(E), E^{\prime}\right)
$$

which takes $f: E \rightarrow \pi_{*} E^{\prime}$ to the induced map $\tilde{f}: P(E) \rightarrow E^{\prime}$ is a bijection. The inverse map takes $g: P(E) \rightarrow E^{\prime}$ to the composite

$$
E \stackrel{\eta}{\rightarrow} \pi_{*} P(E) \stackrel{\pi_{*} g}{\rightarrow} \pi_{*} E^{\prime}
$$

where the right hand map takes $a \in E_{n}^{q}$ to $a[x]^{0} \in P(E)_{n}^{q}$.

Remark 4.2.10. - The proof of [15, Proposition 1.3] shows that

$$
r(f)=F d \lambda\left([f]_{n}\right)-\lambda\left([f]_{n-1}\right)^{p-1} d \lambda\left([f]_{n-1}\right)
$$

is an additive function of $f$. This makes it possible to prove that $r(f)=0$ without the use of Theorem 4.2.8, hence completing a purely algebraic proof of Theorem B.

We conclude with the following result, which we shall need in Section 7 below.

Lemma 4.2.11.-Let $E^{\prime}, E$, and $E^{\prime \prime}$ be Witt complexes and suppose there is a long-exact sequence of strict maps of pro-abelian groups

$$
\cdots \rightarrow E^{\prime q} \stackrel{f^{q}}{\rightarrow} E^{q} \stackrel{g^{q}}{\rightarrow} E_{.}^{\prime \prime q} \stackrel{h^{q}}{\rightarrow} E^{\prime q-1} \rightarrow \cdots
$$

such that the maps commute with $F, d$, and $V$. Then there is an induced long-exact sequence of strict maps of pro-abelian groups

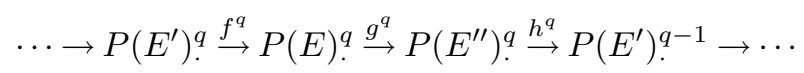

and the maps commute with $F, d$, and $V$.

Proof. - Indeed, as an abelian group $P(E)_{n}^{q}$ is the direct sum of copies of $E_{m}^{q}$ and $E_{m}^{q-1}$ with $1 \leqslant m \leqslant n$. 


\section{The de Rham-Witt complex of Bloch-Deligne-Illusie}

5.1. For $\mathbb{F}_{p}$-algebras, [13] contains a construction of the de Rham-Witt complex that is somewhat more concrete than the construction in Theorem A. In this paragraph we extend Illusie's method to $\mathbb{Z}_{(p)}$-algebras. We recall from [13, I] that a $V$-pro-complex over $A$ consists of:

(i) a pro-differential graded ring $D^{*}$ and a strict map of pro-rings

$$
\lambda: W .(A) \rightarrow D_{.}^{0} ;
$$

(ii) a strict map of pro-graded abelian groups

$$
V: D_{\cdot-1}^{*} \rightarrow D^{*}
$$

such that $\lambda V=V \lambda$ and such that for all $x, y \in D^{*}$ and $a \in A$,

$$
V(x d y)=V(x) d V(y), \quad V(x) d \lambda\left([a]_{n}\right)=V\left(x \lambda\left([a]_{n-1}\right)^{p-1}\right) d V\left(\lambda\left([a]_{n-1}\right)\right) .
$$

A map of $V$-pro-complexes over $A$ is a strict map of pro-differential graded rings $f: D^{*} \rightarrow D^{\prime *}$ such that $\lambda^{\prime}=f \lambda$ and $V^{\prime} f=f V$.

There is a natural forgetful functor from the category of Witt complexes over $A$ to the category of $V$-pro-complexes over $A$,

$$
\mathcal{W}_{A} \rightarrow \mathcal{W}_{A}^{\prime}
$$

Indeed, the calculation

$$
\begin{aligned}
V(x d y) & =V(x F d V y)=V(x) d V(y), \\
V(y) d \lambda\left([a]_{n}\right) & =V\left(y F d \lambda\left([a]_{n}\right)\right)=V\left(y \lambda\left([a]_{n-1}^{p-1}\right) d \lambda\left([a]_{n-1}\right)\right) \\
& =V\left(y \lambda\left([a]_{n-1}^{p-1}\right)\right) d V\left(\lambda\left([a]_{n-1}\right)\right),
\end{aligned}
$$

shows that a Witt complex is a $V$-pro-complex upon forgetting the Frobenius.

The proof of Theorem A shows that the category $\mathcal{W}_{A}^{\prime}$ has an initial object. A more constructive proof is given by Illusie in [13, Theorem I.1.3]. We will need this construction later on, so we include it here.

PROPOSITION 5.1.1. - The category $\mathcal{W}_{A}^{\prime}$ has an initial object $W_{!}^{\prime} \Omega_{A}^{*}$, and the canonical map $\Omega_{W .(A)}^{*} \rightarrow W^{\prime} \Omega_{A}^{*}$ is surjective.

Proof. - One recursively defines the differential graded rings $W_{m}^{\prime} \Omega_{A}^{*}$ and the maps $R, V$, and $\lambda$, starting from $W_{1}^{\prime} \Omega_{A}^{*}=\Omega_{A}^{*}$. So suppose that for all $n<m$, the differential graded ring $W_{n}^{\prime} \Omega_{A}^{*}$ and the maps

$$
R: W_{n}^{\prime} \Omega_{A}^{*} \rightarrow W_{n-1}^{\prime} \Omega_{A}^{*}, \quad V: W_{n-1}^{\prime} \Omega_{A}^{*} \rightarrow W_{n}^{\prime} \Omega_{A}^{*}, \quad \lambda: W_{n}(A) \rightarrow W_{k}^{\prime} \Omega_{A}^{0},
$$

have been constructed such that $R$ is a map of differential graded rings, $V$ is additive, $\lambda R=R \lambda$, $\lambda V=V \lambda$, and such that for all $x, y \in W_{n-1}^{\prime} \Omega_{A}^{*}$ and $a \in A$,

$$
V(x d y)=V(x) d V(y), \quad V(x) d \lambda\left([a]_{n}\right)=V\left(x \lambda\left([a]_{n-1}\right)^{p-1}\right) d V\left(\lambda\left([a]_{n-1}\right)\right) .
$$


Suppose, in addition, that for all $n<m$, the canonical map

$$
\Omega_{W_{n}(A)}^{*} \rightarrow W_{n}^{\prime} \Omega_{A}^{*}
$$

is surjective. Then, one defines

$$
W_{m}^{\prime} \Omega_{A}^{*}=\Omega_{W_{m}(A)}^{*} / N_{m}^{*},
$$

where $N_{m}^{*}$ is the differential graded ideal generated by the elements

$$
\sum_{\alpha} V\left(\lambda\left(x_{\alpha}\right)\right) d V\left(\lambda\left(y_{1, \alpha}\right)\right) \ldots d V\left(\lambda\left(y_{i, \alpha}\right)\right),
$$

for all $x_{\alpha}, y_{i, \alpha} \in W_{m-1}(A)$ such that the sum

$$
\sum_{\alpha} \lambda\left(x_{\alpha}\right) d \lambda\left(y_{1, \alpha}\right) \ldots d \lambda\left(y_{i, \alpha}\right)
$$

is equal to zero in $W_{m-1}^{\prime} \Omega_{A}^{*}$, and by the elements

$$
V(\lambda(x)) d \lambda\left([a]_{m}\right)-V\left(\lambda(x) \lambda\left([a]_{m-1}\right)^{p-1}\right) d V\left(\lambda\left([a]_{m-1}\right)\right),
$$

for all $a \in A$ and $x \in W_{m-1}(A)$. The unique map of differential graded rings

$$
\Omega_{W_{m}(A)}^{*} \rightarrow W_{m-1}^{\prime} \Omega_{A}^{*},
$$

which extends $\lambda R: W_{m}(A) \rightarrow W_{m-1}^{\prime} \Omega_{A}^{0}$, factors to give a map of differential graded rings

$$
R: W_{m}^{\prime} \Omega_{A}^{*} \rightarrow W_{m-1}^{\prime} \Omega_{A}^{*} .
$$

The additive map

$$
V: W_{m-1}^{\prime} \Omega_{A}^{*} \rightarrow W_{m}^{\prime} \Omega_{A}^{*}
$$

given by

$$
V\left(\lambda(x) d \lambda\left(y_{1}\right) \ldots d \lambda\left(y_{i}\right)\right)=V(\lambda(x)) d V\left(\lambda\left(y_{1}\right)\right) \ldots d V\left(\lambda\left(y_{i}\right)\right)
$$

is well-defined and satisfies that $\lambda V=V \lambda$ and that for all $x, y \in W_{m-1}^{\prime} \Omega_{A}^{*}$ and $a \in A$,

$$
V(x d y)=V(x) d V(y), \quad V(x) d \lambda\left([a]_{m}\right)=V\left(x \lambda\left([a]_{m-1}\right)^{p-1}\right) d V\left(\lambda\left([a]_{m-1}\right)\right) .
$$

This gives a $V$-pro-complex $W^{\prime} \Omega_{A}^{*}$. One verifies immediately that this is the initial object in $\mathcal{W}_{A}^{\prime}$.

LEMMA 5.1.4. - The relation $V d=p d V$ holds in $W^{\prime} \Omega_{A}^{*}$.

Proof. - It follows from the construction above that the map $V$ is a map of graded $W_{n}(A)$-modules

$$
V: F_{*} W_{n-1}^{\prime} \Omega_{A}^{*} \rightarrow W_{n}^{\prime} \Omega_{A}^{*},
$$

where on the left, $W_{n-1}^{\prime} \Omega_{A}^{*}$ is considered a $W_{n}(A)$-module via the Frobenius

$$
F: W_{n}(A) \rightarrow W_{n-1}(A)
$$


Hence,

$$
\begin{aligned}
V(d x) & =V(1) d V(x)=d(V(1) V(x))-d V(1) \cdot V(x) \\
& =d V(F V(1) x)-V(d(1) x)=p d V(x) .
\end{aligned}
$$

This proves the lemma.

LEMMA 5.1.5. - Suppose that for the ring A, the canonical map

$$
W^{\prime} \Omega_{A}^{*} \rightarrow W . \Omega_{A}^{*}
$$

is an isomorphism. Then the same is true for $A[x]$.

Proof. - Only the injectivity of the map of the statement is at issue. The assumption of the lemma implies that the induced map

$$
P\left(W^{\prime} \Omega_{A}^{*}\right) \rightarrow P\left(W . \Omega_{A}^{*}\right)
$$

is an isomorphism of pro-graded abelian groups. We proved in Theorem 4.2.8 above that the right hand side is a Witt complex over $A[x]$. Therefore, the left hand side is a $V$-pro-complex over $A[x]$. But then the canonical map

$$
W^{\prime} \Omega_{A[x]}^{*} \rightarrow P\left(W^{\prime} \Omega_{A}^{*}\right)
$$

is an inverse of the map

$$
\tilde{\pi}: P\left(W^{\prime} \Omega_{A}^{*}\right) \rightarrow W^{\prime} \Omega_{A[x]}^{*}
$$

induced from $W^{\prime} \Omega_{A}^{*} \rightarrow \pi_{*} W^{\prime} \Omega_{A[x]}^{*}$.

Proof of Theorem D. - We must construct a map

$$
F: W_{n}^{\prime} \Omega_{A}^{*} \rightarrow W_{n-1}^{\prime} \Omega_{A}^{*}
$$

and show that this makes $W^{\prime} \Omega_{A}^{*}$ a Witt complex over $A$.

Suppose first that $A$ is a polynomial algebra over $\mathbb{Z}_{(p)}$ in a finite number of variables. Then we claim that the canonical map

$$
W^{\prime} \Omega_{A}^{*} \rightarrow W . \Omega_{A}^{*}
$$

is an isomorphism. By Lemma 5.1.5 it suffices to consider the case $A=\mathbb{Z}_{(p)}$. And in this case, the statement follows from Example 1.2.4.

In the general case, we first construct a derivation

$$
\delta: W_{n}(A) \rightarrow W_{n-1}^{\prime} \Omega_{A}^{*}
$$

such that, once $F$ is defined, $\delta=F d \lambda$. Given a Witt vector

$$
a=\left[a_{0}\right]_{n}+V\left(\left[a_{1}\right]_{n-1}\right)+\cdots+V^{n-1}\left(\left[a_{n-1}\right]_{1}\right),
$$

we define

$$
\left.\delta(a)=\lambda\left(\left[a_{0}\right]_{n-1}\right)^{p-1} d \lambda\left(\left[a_{0}\right]_{n-1}\right)+d \lambda\left(\left[a_{1}\right]_{n-1}\right]\right)+\cdots+d V^{n-2}\left(\lambda\left(\left[a_{n-1}\right]_{1}\right)\right) .
$$


In order to verify that $\delta$ is a derivation, we may assume that $A$ is a polynomial algebra over $\mathbb{Z}_{(p)}$ in a finite number of variables. But in this case, the canonical map

$$
W^{\prime} \Omega_{A}^{*} \rightarrow W \cdot \Omega_{A}^{*}
$$

is an isomorphism, and the composite

$$
W_{n}(A) \stackrel{\delta}{\rightarrow} W_{n-1}^{\prime} \Omega_{A}^{*} \stackrel{\sim}{\rightarrow} W_{n-1} \Omega_{A}^{*}
$$

is equal to $F d \lambda$, which is indeed a derivation.

There is a unique map of graded $W_{n}(A)$-algebras

$$
F^{\prime}: \Omega_{W_{n}(A)}^{*} \rightarrow F_{*} W_{n-1}^{\prime} \Omega_{A}^{*}
$$

such that $F^{\prime} d \lambda=\delta: W_{n}(A) \rightarrow W_{n-1}^{\prime} \Omega_{A}^{1}$, and we claim that $F^{\prime}$ annihilates the differential graded ideal $N_{n}^{*}$. Indeed, it follows immediately from the definition of $\delta$ that $\delta(V(a))=d a$, and hence, $F^{\prime}$ annihilates elements of the form (5.1.2). And the calculation

$$
\begin{gathered}
F^{\prime}\left(V(\lambda(x)) d \lambda\left([a]_{n-1}\right)-V\left(\lambda(x) \lambda\left([a]_{n-1}\right)^{p-1}\right) d V\left(\lambda\left([a]_{n-1}\right)\right)\right) \\
=p\left(\lambda(x) \delta\left([a]_{n-1}\right)-\lambda(x) \lambda\left([a]_{n-1}\right)^{p-1} d\left(\lambda\left([a]_{n-1}\right)\right)\right)=0,
\end{gathered}
$$

shows that $F^{\prime}$ annihilates the elements (5.1.3), too. Hence, the map $F^{\prime}$ factors to give a map of graded $W_{n}(A)$-algebras

$$
F: W_{n}^{\prime} \Omega_{A}^{*} \rightarrow W_{n-1}^{\prime} \Omega_{A}^{*} .
$$

It is clear from the way that $F$ was constructed that the canonical map

$$
W^{\prime} \Omega_{A}^{*} \rightarrow W \cdot \Omega_{A}^{*}
$$

commutes with Frobenius operators. The map is an isomorphism, if $A$ is a polynomial algebra in finitely many variables over $\mathbb{Z}_{(p)}$. Hence, in this case, the operator $F$ satisfies the relations which makes $W^{\prime} \Omega_{A}^{*}$ a Witt complex. But then it satisfies these relations for every $\mathbb{Z}_{(p)}$-algebra; compare the proof of 4.2.8.

\section{6. Étale extensions}

6.1. A map of rings $f: A \rightarrow B$, we recall, is étale if it is finitely presented, flat, and if $\Omega_{B / A}^{1}$ vanishes; see [6, $\S 17]$. Let $A$ be a ring in which $p$ is a non-zero-divisor, and let $W_{n, v}(A)$ be the reduction modulo $p^{v}$ of the Witt ring $W_{n}(A)$. We show in Proposition 6.2.2 below that if $A \rightarrow B$ is étale, then the induced map

$$
W_{n, v}(A) \rightarrow W_{n, v}(B)
$$

is étale. The analogous statement for $\mathbb{F}_{p}$-algebras was proved in [13, Proposition 0.1.5.8], and the proof in the case we consider is similar.

We need a slight generalization of standard results about flatness and filtrations, [3, Chapter III, $\S 5]$. Let $A$ be a ring and let $\mathrm{Fil}^{s} A, 0 \leqslant s<n$, be a finite descending filtration by ideals,

$$
A=\mathrm{Fil}^{0} A \supset \mathrm{Fil}^{1} A \supset \cdots \supset \mathrm{Fil}^{n} A=0 .
$$


The filtration is called multiplicative if for $0 \leqslant s, t<n$, the multiplication maps

$$
\mathrm{Fil}^{s} A \otimes_{A} \mathrm{Fil}^{t} A \rightarrow \mathrm{Fil}^{s+t} A .
$$

If $N$ is an $A$-module, we have the induced filtration $\mathrm{Fil}^{s} N, 0 \leqslant s<n$, where $\mathrm{Fil}^{s} N$ is the image of the canonical map Fil ${ }^{s} A \otimes_{A} N \rightarrow N$.

LEMMA 6.1.1. - Let $\mathrm{Fil}^{s} A, 0 \leqslant s<n$, be a finite descending multiplicative filtration of the ring $A$, and let $M$ be an A-module. Suppose that $\mathrm{gr}^{0} M$ is a flat $\mathrm{gr}^{0} A$-module and that the canonical map

$$
\mathrm{Fil}^{1} A \otimes_{A} M \rightarrow \mathrm{Fil}^{1} M
$$

is an isomorphism. Then $M$ is a flat A-module.

Proof. - The sequence

$$
\operatorname{Tor}_{1}^{A}(A, M) \rightarrow \operatorname{Tor}_{1}^{A}\left(\operatorname{gr}^{0} A, M\right) \rightarrow \operatorname{Fil}^{1} A \otimes_{A} M \rightarrow M
$$

shows that $\operatorname{Tor}_{1}^{A}\left(\operatorname{gr}^{0} A, M\right)$ vanishes. Since $\operatorname{gr}^{0} M$ is assumed $\operatorname{gr}^{0} A$-flat, this implies that $\operatorname{Tor}_{1}^{A}(N, M)$ vanishes for every $A$-module $N$ which is annihilated by $\mathrm{Fil}^{1} A$. Indeed, the change of rings spectral sequence

$$
E_{s, t}^{2}=\operatorname{Tor}_{p}^{\mathrm{gr}^{0} A}\left(N, \operatorname{Tor}_{q}^{A}\left(\operatorname{gr}^{0} A, M\right)\right) \Rightarrow \operatorname{Tor}_{s+t}^{A}(N, M)
$$

has vanishing $E^{2}$-term in total degree one. In general, the short exact sequences

$$
0 \rightarrow \mathrm{Fil}^{s+1} N \rightarrow \mathrm{Fil}^{s} N \rightarrow \mathrm{gr}^{s} N \rightarrow 0
$$

give rise to exact sequences

$$
\operatorname{Tor}_{1}^{A}\left(\mathrm{Fil}^{s+1} N, M\right) \rightarrow \operatorname{Tor}_{1}^{A}\left(\mathrm{Fil}^{s} N, M\right) \rightarrow \operatorname{Tor}_{1}^{A}\left(\mathrm{gr}^{s} N, M\right)
$$

The right hand term vanishes by the above, since the module $\operatorname{gr}^{s} N$ is annihilated by $\mathrm{Fil}^{1} A$. But Fil $^{n} N$ is zero, and hence by easy induction, $\operatorname{Tor}_{1}^{A}(N, M)$ vanishes. Thus $M$ is a flat $A$-module.

Lemma 6.1.2. - Let $\mathrm{Fil}^{s} A, 0 \leqslant s<n$, be a finite descending multiplicative filtration of the ring $A$, and let $M$ be an A-module. Suppose that for $0 \leqslant s<n$, the canonical map

$$
\operatorname{gr}^{s} A \otimes \operatorname{gr}^{0} A \operatorname{gr}^{0} M \rightarrow \operatorname{gr}^{s} M
$$

is an isomorphism. Then $\mathrm{Fil}^{s} A \otimes_{A} M \stackrel{\sim}{\rightarrow} \mathrm{Fil}^{s} M$ is an isomorphism, $0 \leqslant s<n$.

Proof. - The assumptions imply that the canonical map

$$
\operatorname{gr}^{s} A \otimes_{A} M \rightarrow \operatorname{gr}^{s} M
$$

is an isomorphism. Indeed, the left hand map in the exact sequence

$$
\mathrm{gr}^{s} A \otimes_{A} \mathrm{Fil}^{1} M \rightarrow \mathrm{gr}^{s} A \otimes_{A} M \rightarrow \mathrm{gr}^{s} A \otimes_{A} \operatorname{gr}^{0} M \rightarrow 0
$$


is zero. The statement now follows from the diagram

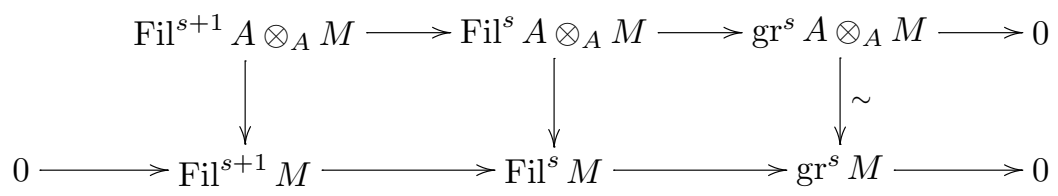

by induction, starting from $s=n-1$.

LeMma 6.1.3. - Let $f: A \rightarrow B$ be a ring homomorphism, let $I \subset A$ be a nilpotent ideal, and suppose that $\Omega_{(B / I B) /(A / I)}^{1}$ vanishes. Then $\Omega_{B / A}^{1}$ vanishes.

Proof. - In the short-exact sequence

$$
0 \rightarrow I \Omega_{B / A}^{1} \rightarrow \Omega_{B / A}^{1} \rightarrow \Omega_{B / A}^{1} \otimes_{A} A / I \rightarrow 0
$$

the right hand term is isomorphic to $\Omega_{\left(B \otimes_{A} A / I\right) /(A / I)}^{1}$, which vanishes by assumption. Hence, the left hand map is an isomorphism. By simple induction, so is

$$
I^{n} \Omega_{B / A}^{1} \stackrel{\sim}{\rightarrow} \Omega_{B / A}^{1},
$$

for all $n \geqslant 0$, and since $I$ is nilpotent, $\Omega_{B / A}^{1}$ is zero.

6.2. If $p$ is a non-zero-divisor in $A$ and if $f: A \rightarrow B$ is flat, then $p$ is a non-zero-divisor in $B$. Indeed, this follows from the diagram

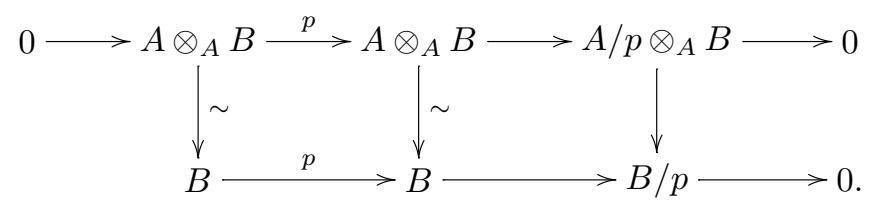

We recall from [7, XIV, $\S 1$, Proposition 2] that if $f: A \rightarrow B$ is an étale map of $\mathbb{F}_{p}$-algebras, then the following diagram, where $\varphi$ is the Frobenius, is cocartesian.

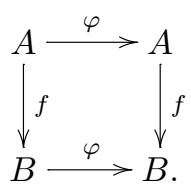

This means that we can write every $b \in B$ as a sum

$$
b=\sum_{i} b_{i}^{p} f\left(a_{i}\right)
$$

with $b_{i} \in B$ and $a_{i} \in A$. 
Proposition 6.2.2. - Let $f: A \rightarrow B$ be an étale map and suppose that $p$ is a non-zerodivisor in $A$. Then for all $n, v \geqslant 1$ and all $0 \leqslant s<n, W_{n, v}(f)$ is étale and the diagrams
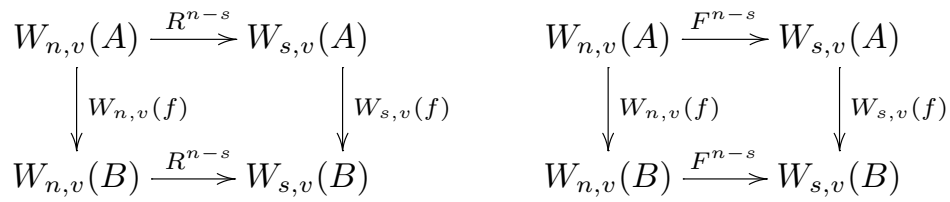

are cocartesian in the category of commutative rings.

Proof. - Suppose first that $v=1$. The $V$-filtrations of $W_{n, 1}(A)$ and $W_{n, 1}(B)$ are finite and multiplicative. But in order to apply the results of the previous section, we must first show that the $V$-filtration of $W_{n, 1}(B)$ is equal to the filtration induced by the $V$-filtration of $W_{n, 1}(A)$, or equivalently, that the canonical map

$$
W_{n, 1}(B) \otimes_{W_{n, 1}(A)} V^{s} W_{n, 1}(A) \rightarrow V^{s} W_{n, 1}(B)
$$

is surjective. This, we note, is equivalent to the statement that the left hand square in the statement of the proposition is cocartesian. Indeed, there is a natural short-exact sequence of $W_{n, v}(A)$ modules

$$
0 \rightarrow F_{*}^{s} W_{n-s, 1}(A) \stackrel{V^{s}}{\rightarrow} W_{n, 1}(A) \stackrel{R^{n-s}}{\longrightarrow} R_{*}^{n-s} W_{s, 1}(A) \rightarrow 0,
$$

and the left hand map has image $V^{s} W_{n, 1}(A)$. In particular, it will suffice to consider the case $s=n-1$. But the map

$$
W_{n, 1}(B) \otimes_{W_{n, 1}(A)} F_{*}^{n-1} A_{1} \rightarrow F_{*}^{n-1} B_{1}
$$

takes $[b]_{n} \otimes a$ to $b^{p^{n-1}} f(a)$, and hence, is surjective by (6.2.1). Indeed, $f_{1}: A_{1} \rightarrow B_{1}$ is étale since $f: A \rightarrow B$ is. Hence, the $V$-filtration of $W_{n, 1}(B)$ is equal to the filtration induced from the $V$-filtration of $W_{n, 1}(A)$. We can now conclude from Lemma 6.1.2 that the canonical map

$$
W_{n, 1}(B) \otimes_{W_{n, 1}(A)} V^{s} W_{n, 1}(A) \rightarrow V^{s} W_{n, 1}(B)
$$

is an isomorphism, or equivalently, that the right hand square in the statement of the proposition is cocartesian (with $s$ and $n-s$ interchanged). Indeed, the map

$$
\operatorname{gr}_{V}^{s} W_{n, 1}(A) \otimes_{\operatorname{gr}_{V}^{0} W_{n, 1}(A)} \operatorname{gr}_{V}^{0} W_{n, 1}(B) \rightarrow \operatorname{gr}_{V}^{s} W_{n, 1}(B)
$$

is naturally identified with the canonical map

$$
\varphi_{*}^{s} A_{1} \otimes_{A_{1}} B_{1} \rightarrow \varphi_{*}^{s} B_{1},
$$

and the latter is an isomorphism by (6.2.1).

We can now show that $W_{n, 1}(f)$ is étale. First, $W_{n, 1}(f)$ is finitely presented. To see this, it suffices to show that $\operatorname{gr}_{V} W_{n, 1}(f)$ is finitely presented. But this follows from the isomorphism

$$
B_{1} \otimes_{A_{1}} \operatorname{gr}_{V} W_{n, 1}(A) \stackrel{\sim}{\rightarrow} \operatorname{gr}_{V}^{\cdot} W_{n, 1}(B),
$$


since $f_{1}: A_{1} \rightarrow B_{1}$ is finitely presented. Next, it follows from Lemma 6.1.1 that $W_{n, 1}(f)$ is flat; for $f_{1}: A_{1} \rightarrow B_{1}$ is flat and the canonical map

$$
W_{n, 1}(B) \otimes_{W_{n, 1}(A)} V W_{n, 1}(A) \rightarrow V W_{n, 1}(B)
$$

is an isomorphism. Finally, since $V W_{n, 1}(A) \subset W_{n, 1}(A)$ is a square-zero ideal, and since $\Omega_{B_{1} / A_{1}}^{1}$ vanishes, Lemma 6.1.3 shows that $\Omega_{W_{n, 1}(B) / W_{n, 1}(A)}^{1}$ is zero. This completes the proof of the proposition, if $v=1$.

In the general case $v \geqslant 1$, we consider the $p$-adic filtration of $W_{n, v}(A)$, which is finite and multiplicative. Moreover, the canonical map

$$
p^{s} W_{n, v}(A) \otimes_{W_{n, v}(A)} W_{n, v}(B) \rightarrow p^{s} W_{n, v}(B),
$$

clearly, is an isomorphism. Hence, one can easily conclude from the case $v=1$ that $W_{n, v}(f)$ is étale. It remains to prove that the two squares in the statement of the proposition are cocartesian. As we noted earlier, this is equivalent to the statement that for all $0 \leqslant s<n$, the canonical map

$$
V^{s} W_{n, v}(A) \otimes_{W_{n, v}(A)} W_{n, v}(B) \rightarrow V^{s} W_{n, v}(B)
$$

is an isomorphism. Injectivity follows immediately from the fact that $W_{n, v}(f)$ is flat. For surjectivity it suffices to prove the case $s=n-1$. It follows by induction from (6.2.1) that every $b \in B$ can be written

$$
b=\sum a_{i} b_{i}^{p^{n-1}}+p^{v} b^{\prime}
$$

with $a_{i} \in A$ and $b_{i}, b^{\prime} \in B$. Hence

$$
V^{n-1}(b)=\sum V^{n-1}\left(a_{i}\right)\left[b_{i}\right]+p^{v} V^{n-1}\left(b^{\prime}\right),
$$

which proves surjectivity.

Proposition 6.2.3. - Let $A \rightarrow B$ be an étale map and suppose that $p$ is a non-zero-divisor in $A$. Then for all $n, v \geqslant 1$ and $q \geqslant 0$, the canonical map

$$
W_{n, v}(B) \otimes_{W_{n, v}(A)} W_{n, v} \Omega_{A}^{q} \rightarrow W_{n, v} \Omega_{B}^{q}
$$

is an isomorphism.

Proof. - This is proved from Proposition 6.2.2 by the argument of [13, Proposition I.1.14]: to produce the inverse of the map of the statement one shows that the left hand side is a $V$-procomplex.

Proposition 6.2.4. - Let $f: A \rightarrow B$ be an étale map and suppose that $p$ is a non-zerodivisor in $A$. Then for all $n, v \geqslant 1$ and $q \geqslant 0$, the canonical map

$$
W_{n, v}(B) \otimes_{W_{n, v}(A)} \operatorname{TR}_{q}^{n}\left(A ; p, \mathbb{Z} / p^{v}\right) \rightarrow \operatorname{TR}_{q}^{n}\left(B ; p, \mathbb{Z} / p^{v}\right)
$$

is an isomorphism.

Proof. - The proof is by induction on $n$ starting from the case $n=1$, which was proved in [5, Proposition 3.2.1]. The proof of the induction step is similar to the proof of [10, Theorem 5.5]. 
In brief, there is a natural long exact sequence of $W_{n, v}(A)$-modules

$$
\cdots \rightarrow{ }_{h} \mathrm{TR}_{q}^{n}\left(A ; p, \mathbb{Z} / p^{v}\right) \rightarrow \mathrm{TR}_{q}^{n}\left(A ; p, \mathbb{Z} / p^{v}\right) \stackrel{R}{\rightarrow} R_{*} \operatorname{TR}_{q}^{n-1}\left(A ; p, \mathbb{Z} / p^{v}\right) \rightarrow \cdots
$$

The base-change of this sequence along $W_{n, v}(f)$, which is exact since $W_{n, v}(f)$ is flat, maps to the long-exact sequence of $W_{n, v}(B)$-modules

$$
\cdots \rightarrow{ }_{h} \operatorname{TR}_{q}^{n}\left(B ; p, \mathbb{Z} / p^{v}\right) \rightarrow \operatorname{TR}_{q}^{n}\left(B ; p, \mathbb{Z} / p^{v}\right) \stackrel{R}{\rightarrow} R_{*} \operatorname{TR}_{q}^{n-1}\left(B ; p, \mathbb{Z} / p^{v}\right) \rightarrow \cdots .
$$

The map of the right hand terms,

$$
W_{n, v}(B) \otimes_{W_{n, v}(A)} R_{*} \operatorname{TR}_{q}^{n-1}\left(A ; p, \mathbb{Z} / p^{v}\right) \rightarrow R_{*} \operatorname{TR}_{q}^{n-1}\left(B ; p, \mathbb{Z} / p^{v}\right),
$$

inductively, is an isomorphism, since the left hand square in the statement of Proposition 6.2.2 is cocartesian. In order to show that the map of left hand terms,

$$
W_{n, v}(B) \otimes_{W_{n, v}(A) h} \operatorname{TR}_{q}^{n}\left(A ; p, \mathbb{Z} / p^{v}\right) \rightarrow{ }_{h} \operatorname{TR}_{q}^{n}\left(B ; p, \mathbb{Z} / p^{v}\right),
$$

is an isomorphism, we recall that there is a natural first quadrant spectral sequence of $W_{n, v}(A)$-modules

$$
E_{s, t}^{2}=H_{s}\left(C_{p^{n}}, F_{*}^{n-1} \operatorname{TR}_{*}^{1}\left(A ; p, \mathbb{Z} / p^{v}\right)\right) \Rightarrow_{h} \operatorname{TR}_{s+t}^{n}\left(A ; p, \mathbb{Z} / p^{v}\right) ;
$$

see the discussion preceding [10, Theorem 5.5] and also [9, §4]. The desired isomorphism now follows from the case $n=1$, since the left hand square in the statement of Proposition 6.2.2 is cocartesian.

\section{Smooth $V$-algebras}

7.1. In this paragraph we prove Theorem $\mathrm{E}$ of the introduction. Let $V$ be a complete discrete valuation ring of mixed characteristic $(0, p)$ with quotient field $K$ and perfect residue field $k$. Let $A$ be a smooth $V$-algebra, let $A_{K}=A \otimes_{V} K$, and let $A_{k}=A \otimes_{V} k$.

Lemma 7.1.1. - Let $A$ be a smooth $V$-algebra and let $f: A \rightarrow B$ be an étale map. Then the canonical map is an isomorphism:

$$
W_{n, v}(B) \otimes_{W_{n, v}(A)} \operatorname{TR}_{q}^{n}\left(A \mid A_{K} ; p, \mathbb{Z} / p^{v}\right) \stackrel{\sim}{\rightarrow} \operatorname{TR}_{q}^{n}\left(B \mid B_{K} ; p, \mathbb{Z} / p^{v}\right) .
$$

Proof. - We recall from Proposition 6.2.4 that the canonical map

$$
W_{n, v}(B) \otimes_{W_{n, v}(A)} \operatorname{TR}_{q}^{n}\left(A ; p, \mathbb{Z} / p^{v}\right) \rightarrow \operatorname{TR}_{q}^{n}\left(B ; p, \mathbb{Z} / p^{v}\right)
$$

is an isomorphism. We proved in [9, Remark 1.5.8] that there is a long-exact sequence of $W_{n, v}(A)$-modules

$$
\cdots \rightarrow \mathrm{TR}_{q}^{n}\left(A_{k} ; p, \mathbb{Z} / p^{v}\right) \stackrel{i^{!}}{\rightarrow} \mathrm{TR}_{q}^{n}\left(A ; p, \mathbb{Z} / p^{v}\right) \stackrel{j_{*}}{\rightarrow} \mathrm{TR}_{q}^{n}\left(A \mid A_{K} ; p, \mathbb{Z} / p^{v}\right) \rightarrow \cdots
$$

where the left hand term is a $W_{n, v}(A)$-module via $i_{*}: W_{n, v}(A) \rightarrow W_{n, v}\left(A_{k}\right)$. We claim that also the canonical map

$$
W_{n, v}(B) \otimes_{W_{n, v}(A)} \operatorname{TR}_{q}^{n}\left(A_{k} ; p, \mathbb{Z} / p^{v}\right) \rightarrow \operatorname{TR}_{q}^{n}\left(B_{k} ; p, \mathbb{Z} / p^{v}\right)
$$


is an isomorphism. Since $W_{n, v}(A) \rightarrow W_{n, v}(B)$ is flat by Proposition 6.2.2, the obvious fivelemma argument completes the proof. To prove the claim, we first recall from [8, Proposition 2.4.4] that the canonical map

$$
W_{n}\left(B_{k}\right) \otimes_{W_{n}\left(A_{k}\right)} \operatorname{TR}_{q}^{n}\left(A_{k} ; p\right) \rightarrow \mathrm{TR}_{q}^{n}\left(B_{k} ; p\right)
$$

is an isomorphism; the proof is analogous to the proof of Proposition 6.2.4 above. A five-lemma argument based on the coefficient sequence

$$
\cdots \rightarrow \mathrm{TR}_{q}^{n}\left(A_{k} ; p\right) \stackrel{p^{v}}{\rightarrow} \mathrm{TR}_{q}^{n}\left(A_{k} ; p\right) \rightarrow \mathrm{TR}_{q}^{n}\left(A_{k} ; p, \mathbb{Z} / p^{v}\right) \stackrel{\beta}{\rightarrow} \mathrm{TR}_{q-1}^{n}\left(A_{k} ; p\right) \rightarrow \cdots
$$

shows that the canonical map

$$
W_{n, v}\left(B_{k}\right) \otimes_{W_{n, v}\left(A_{k}\right)} \operatorname{TR}_{q}^{n}\left(A_{k} ; p, \mathbb{Z} / p^{v}\right) \rightarrow \mathrm{TR}_{q}^{n}\left(B_{k} ; p, \mathbb{Z} / p^{v}\right)
$$

is an isomorphism. Hence, it suffices to show that

$$
W_{n, v}(B) \otimes_{W_{n, v}(A)} W_{n, v}\left(A_{k}\right) \rightarrow W_{n, v}\left(B_{k}\right)
$$

is an isomorphism. The statement for $v$ implies the statement for $v-1$, so we can assume that $n \leqslant v$. Then $W_{n, v}\left(A_{k}\right)=W_{n}\left(A_{k}\right)$ and $W_{n, v}\left(B_{k}\right)=W_{n}\left(B_{k}\right)$. We proceed by induction on $1 \leqslant n \leqslant v$ starting from the trivial case $n=1$. In the induction step, we consider the short exact sequence of $W_{n, v}(A)$-modules

$$
0 \rightarrow F_{*}^{n-1} A_{k} \stackrel{V^{n-1}}{\longrightarrow} W_{n}\left(A_{k}\right) \stackrel{R}{\longrightarrow} R_{*} W_{n-1}\left(A_{k}\right) \rightarrow 0
$$

(the corresponding sequence for $W_{n, v}\left(A_{k}\right)$ is not exact, if $\left.v<n\right)$. We wish to show that the upper horizontal map in the diagram

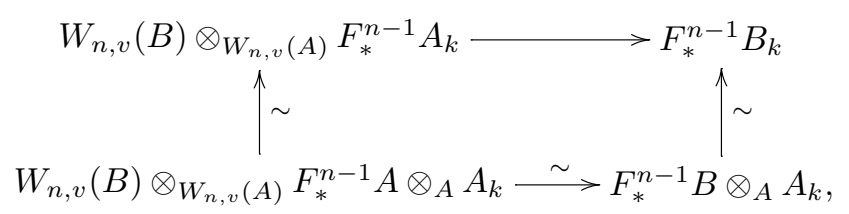

is an isomorphism. But Proposition 6.2.2 shows that the lower horizontal map is an isomorphism, and the vertical maps are isomorphisms for trivial reasons. One shows in a similar fashion that the map

$$
W_{n, v}(B) \otimes_{W_{n, v}(A)} R_{*} W_{n-1}\left(A_{k}\right) \stackrel{\sim}{\rightarrow} R_{*} W_{n-1}\left(B_{k}\right)
$$

is an isomorphism. This completes the proof.

We recall from the introduction that $W \cdot \Omega_{\left(A, M_{A}\right)}^{*}$ denotes the universal Witt complex over the $\log \operatorname{ring}\left(A, M_{A}\right)$; see also [9, Section 3.2].

Lemma 7.1.2. - Let $A$ be a smooth $V$-algebra and let $f: A \rightarrow B$ be an étale map. Then the canonical map is an isomorphism:

$$
W_{n, v}(B) \otimes_{W_{n, v}(A)} W_{n, v} \Omega_{\left(A, M_{A}\right)}^{q} \stackrel{\sim}{\rightarrow} W_{n, v} \Omega_{\left(B, M_{B}\right)}^{q} .
$$


Proof. - This is similar to the proof of Proposition 6.2.3.

Lemma 7.1.3. - Let A be a smooth $V$-algebra. Then the canonical map

$$
P\left(\mathrm{TR}_{*}\left(A \mid A_{K} ; p, \mathbb{Z} / p^{v}\right)\right) \rightarrow \mathrm{TR}_{*}\left(A[x] \mid A[x]_{K} ; p, \mathbb{Z} / p^{v}\right)
$$

is an isomorphism.

Proof. - By Theorem C, the canonical map

$$
P\left(\mathrm{TR}_{*}(R ; p)\right) \rightarrow \mathrm{TR}_{*}(R[x] ; p)
$$

is an isomorphism, for every $\mathbb{Z}_{(p)}$-algebra $R$. The coefficient sequence

$$
\cdots \rightarrow \mathrm{TR}_{q}^{n}(R ; p) \stackrel{p^{v}}{\rightarrow} \mathrm{TR}_{q}^{n}(R ; p) \rightarrow \mathrm{TR}_{q}^{n}\left(R ; p, \mathbb{Z} / p^{v}\right) \stackrel{\beta}{\rightarrow} \mathrm{TR}_{q-1}^{n}(R ; p) \rightarrow \cdots
$$

by Lemma 4.2.11, gives rise to a long-exact sequence

$$
\cdots \rightarrow P\left(\mathrm{TR}_{*}(R ; p)\right)_{q}^{n} \stackrel{p^{v}}{\rightarrow} P\left(\mathrm{TR}_{*}(R ; p)\right)_{q}^{n} \rightarrow P\left(\mathrm{TR}_{*}\left(R ; p, \mathbb{Z} / p^{v}\right)\right)_{q}^{n} \rightarrow \cdots
$$

which maps to the coefficient sequence

$$
\cdots \rightarrow \mathrm{TR}_{q}^{n}(R[x] ; p) \stackrel{p^{v}}{\rightarrow} \mathrm{TR}_{q}^{n}(R[x] ; p) \rightarrow \mathrm{TR}_{q}^{n}\left(R[x] ; p, \mathbb{Z} / p^{v}\right) \rightarrow \cdots .
$$

By Theorem $\mathrm{C}$, this map is an isomorphism of two out of three terms, and hence, of the remaining terms. This shows that for every $\mathbb{Z}_{(p)}$-algebra $R$, the canonical map

$$
P\left(\mathrm{TR}_{*}\left(R ; p, \mathbb{Z} / p^{v}\right)\right) \rightarrow \mathrm{TR}_{*}\left(R[x] ; p, \mathbb{Z} / p^{v}\right)
$$

is an isomorphism. This applies, in particular, to $R=A$ and $R=A_{k}$. A similar argument based on the sequence

$$
\cdots \rightarrow \mathrm{TR}_{q}^{n}\left(A_{k} ; p, \mathbb{Z} / p^{v}\right) \stackrel{i^{!}}{\rightarrow} \operatorname{TR}_{q}^{n}\left(A ; p, \mathbb{Z} / p^{v}\right) \stackrel{j_{*}}{\rightarrow} \operatorname{TR}_{q}^{n}\left(A \mid A_{K} ; p, \mathbb{Z} / p^{v}\right) \rightarrow \cdots
$$

completes the proof.

Lemma 7.1.4. - Let A be a V-algebra. Then the canonical map

$$
P\left(W . \Omega_{\left(A, M_{A}\right)}^{*} \otimes_{\mathbb{Z}} S_{\mathbb{Z} / p^{v}}\left(\mu_{p^{v}}\right)\right) \rightarrow W \cdot \Omega_{\left(A[x], M_{A[x]}\right)}^{*} \otimes_{\mathbb{Z}} S_{\mathbb{Z} / p^{v}}\left(\mu_{p^{v}}\right)
$$

is an isomorphism.

Proof. - Let $\pi: A \rightarrow A[x]$ be the inclusion of the constant polynomials. A functor which has a right adjoint preserves colimits; in particular, it preserves initial objects. It follows that the canonical map

$$
W \cdot \Omega_{\left(A[x], M_{A[x]}\right)}^{*} \rightarrow \pi^{*} W \cdot \Omega_{\left(A, M_{A}\right)}^{*}
$$

is an isomorphism. Hence, by Theorem $\mathrm{B}$, we have a canonical isomorphism

$$
W \cdot \Omega_{\left(A[x], M_{A[x]}\right)}^{*} \stackrel{\sim}{\rightarrow} P\left(W . \Omega_{\left(A, M_{A}\right)}^{*}\right) .
$$


Finally, the canonical map

$$
P\left(W \cdot \Omega_{\left(A, M_{A}\right)}^{*}\right) \otimes_{\mathbb{Z}} S_{\mathbb{Z} / p^{v}}\left(\mu_{p^{v}}\right) \rightarrow P\left(W \cdot \Omega_{\left(A, M_{A}\right)}^{*} \otimes_{\mathbb{Z}} S_{\mathbb{Z} / p^{v}}\left(\mu_{p^{v}}\right)\right)
$$

is an isomorphism, since $F$ (respectively $d$ ) is the identity map (respectively the zero map) on the factor $S_{\mathbb{Z} / p^{v}}\left(\mu_{p^{v}}\right)$. For instance,

$$
V^{s}(\omega) \otimes \zeta=V^{s}\left(\omega \otimes F^{s}(\zeta)\right)=V^{s}(\omega \otimes \zeta)
$$

The lemma follows.

A pro-abelian group $D$ is Mittag-Leffler zero, if for all $n \geqslant 1$, there exists $m \geqslant n$ such that the structure map $D_{m} \rightarrow D_{n}$ is zero. A strict map $f: D \rightarrow D^{\prime}$ of pro-abelian groups is an isomorphism of pro-abelian groups if and only if the kernel and cokernel of $f$ are Mittag-Leffler zero.

LEMMA 7.1.5. - Let $f: E \rightarrow E^{\prime}$ be a map of Witt complexes and suppose that, as a map of pro-abelian groups, $f$ is an isomorphism. Then, as a map of pro-abelian groups,

$$
P(f): P(E) \rightarrow P\left(E^{\prime}\right)
$$

is an isomorphism.

Proof. - Let $K$ be the kernel of $f: E \rightarrow E^{\prime}$ considered as a strict map of pro-abelian groups, and, by slight abuse of notation, let $P(K)$ denote the kernel of $P(f): P(E) \rightarrow P\left(E^{\prime}\right)$ considered as a strict map of pro-abelian groups. For $n \geqslant 1$, we can find $t \geqslant 0$ such that for all $1 \leqslant s \leqslant n$, the structure map $E_{s+t} \rightarrow E_{s}$ is equal to zero. By inspection, we see that the structure map $P(K)_{n+t} \rightarrow P(K)_{n}$ is zero, and hence, $P(K)$ is Mittag-Leffler zero. A similar argument shows that also the cokernel of $P(f): P(E) \rightarrow P\left(E^{\prime}\right)$ is Mittag-Leffler zero.

Proof of Theorem E. - We recall from [6, Corollary 17.11.4] that a $V$-algebra $A$ is smooth if and only if there exist relatively prime elements $f_{1}, \ldots, f_{r} \in A$ and étale maps

$$
V\left[x_{1}, \ldots, x_{n}\right] \rightarrow A_{f_{i}}=A\left[\frac{1}{f_{i}}\right]
$$

We first prove the statement for polynomial algebras. The proof is by induction on the number of variables; the basic case $A=V$ is the statement of [9, Theorem C]. In the induction step, we assume the statement for $A$ and consider the following diagram of pro-abelian groups.

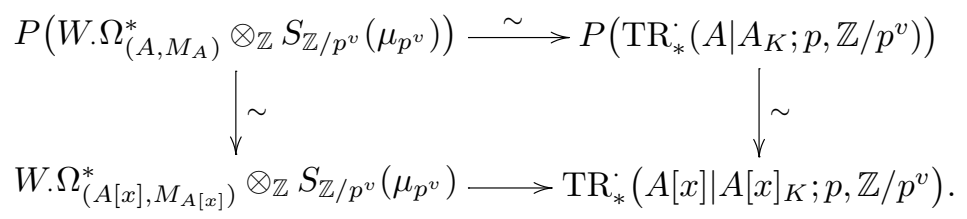

The left and right hand vertical maps are isomorphism by Lemmas 7.1.4 and 7.1.3, respectively, and the top horizontal map is an isomorphism by Lemma 7.1.5 and by the assumption that the theorem holds for $A$. This proves the induction step.

Let $A$ be a smooth $V$-algebra, let $f: A \rightarrow B$ be an étale map, and suppose that the theorem holds for $A$ such that the canonical map

$$
W . \Omega_{\left(A, M_{A}\right)}^{*} \otimes_{\mathbb{Z}} S_{\mathbb{Z} / p^{v}}\left(\mu_{p^{v}}\right) \rightarrow \mathrm{TR}_{*}\left(A \mid A_{K} ; p, \mathbb{Z} / p^{v}\right)
$$


is an isomorphism of pro-graded $W_{\cdot, v}(A)$-modules. Then the map obtained by base-change along $W_{\cdot, v}(f)$ again is an isomorphism, and hence, Lemmas 7.1.2 and 7.1.1 show that the canonical map

$$
W . \Omega_{\left(B, M_{B}\right)}^{*} \otimes_{\mathbb{Z}} S_{\mathbb{Z} / p^{v}}\left(\mu_{p^{v}}\right) \rightarrow \mathrm{TR}_{*}\left(B \mid B_{K} ; p, \mathbb{Z} / p^{v}\right)
$$

is an isomorphism.

The proof is completed by the following covering argument. Let $E_{n}$ be a functor, which to a smooth $V$-algebra $A$ associates a $W_{n, v}(A)$-module $E_{n}(A)$, and suppose that for all $f \in A$, the canonical map

$$
W_{n, v}\left(A_{f}\right) \otimes_{W_{n, v}(A)} E_{n}(A) \rightarrow E_{n}\left(A_{f}\right)
$$

is an isomorphism. Let $f_{1}, \ldots, f_{r} \in A$ be relatively prime elements. Then the canonical map $A \rightarrow \prod_{1 \leqslant i \leqslant r} A_{f_{i}}$ is faithfully flat, and hence, the Koszul complex

$$
C^{*}=\bigotimes_{1 \leqslant i \leqslant r}\left(A \rightarrow A_{f_{i}}\right)
$$

is exact. Here we view $A \rightarrow A_{f_{i}}$ as a cochain complex of $A$-modules with $A$ located in degree zero. The maps in this complex are alternating sums of $A$-algebra homomorphisms. Hence, we obtain complexes $W_{n, v}\left(C^{*}\right)$ and $E_{n}\left(C^{*}\right)$ by applying $W_{n, v}(-)$ and $E_{n}(-)$, respectively, to each term of the complex $C^{*}$. The former complex is exact by an induction argument based on the natural exact sequences

$$
0 \rightarrow W_{1, v}\left(A_{f}\right) \stackrel{V^{n-1}}{\longrightarrow} W_{n, v}\left(A_{f}\right) \stackrel{R}{\longrightarrow} W_{n-1, v}\left(A_{f}\right) \rightarrow 0 .
$$

The terms of this complex are flat $W_{n, v}(A)$-modules by Proposition 6.2.2. Hence, also the common complex

$$
W_{n, v}\left(C^{*}\right) \otimes_{W_{n, v}(A)} E_{n}(A) \stackrel{\sim}{\rightarrow} E_{n}\left(C^{*}\right)
$$

is exact. We now consider the map of Witt complexes $E^{*} \rightarrow E^{\prime *}$ from the statement of Theorem E. Then the complexes $E^{q}\left(C^{*}\right)$ and $E_{.}^{\prime q}\left(C^{*}\right)$ are exact, for all $q \geqslant 0$, by Lemmas 7.1.2 and 7.1.1, respectively. We can choose $f_{1}, \ldots, f_{r} \in A$ such that the map of cochain complexes $E^{q}\left(C^{*}\right) \rightarrow E^{\prime q}\left(C^{*}\right)$ is an isomorphism in positive degrees. But then this map is an isomorphism also in degree zero.

\section{REFERENCES}

[1] BLoch S., Algebraic K-theory and crystalline cohomology, Inst. Hautes Études Sci. Publ. Math. 47 (1977) 187-268.

[2] Bökstedt M., Hsiang W.-C., MAdsen I., The cyclotomic trace and algebraic $K$-theory of spaces, Invent. Math. 111 (1993) 465-540.

[3] Bourbaki N., Commutative Algebra, Chapters 1-7, in: Elements of Mathematics, Springer-Verlag, Berlin, 1998. Translated from the French. Reprint of the 1989 English translation.

[4] Geisser T., Hesselholt L., The de Rham-Witt complex and $p$-adic vanishing cycles, Preprint, 2003.

[5] Geisser T., Hesselholt L., Topological cyclic homology of schemes, in: K-Theory (Seattle, 1997), in: Proc. Symp. Pure Math., vol. 67, 1999, pp. 41-87.

[6] Grothendieck A., Éléments de géométrie algébrique. IV. Étude locale des schémas et des morphismes de schémas (Quatrième Partie), Inst. Hautes Études Sci. Publ. Math. 32 (1967). 
[7] Grothendieck A., Cohomologie $\chi$-adique et fonctions L, in: Lecture Notes in Math., vol. 589, Springer-Verlag, 1977.

[8] Hesselholt L., On the p-typical curves in Quillen's K-theory, Acta Math. 177 (1997) 1-53.

[9] Hesselholt L., Madsen I., On the K-theory of local fields, Ann. of Math. 158 (2003) 1-113.

[10] Hesselholt L., Madsen I., On the $K$-theory of finite algebras over Witt vectors of perfect fields, Topology 36 (1997) 29-102.

[11] Hovey M., Shipley B., Smith J., Symmetric spectra, J. Amer. Math. Soc. 13 (2000) 149-208.

[12] Hyodo O., KATo K., Semi-stable reduction and crystalline cohomology with logarithmic poles, in: Périodes p-adiques (Bures-sur-Yvette, 1988), in: Astérisque, vol. 223, 1994, pp. 221-268.

[13] Illusie L., Complexe de de Rham-Witt et cohomologie cristalline, Ann. Sci. École Norm. Sup. 12 (4) (1979) 501-661.

[14] Kato K., Logarithmic structures of Fontaine-Illusie, in: Algebraic Analysis, Geometry, and Number Theory, Proceedings of the JAMI Inaugural Conference (Baltimore, 1988), Johns Hopkins Univ. Press, Baltimore, MD, 1989, pp. 191-224.

[15] LANGER A., ZINK T., De Rham-Witt cohomology for a proper and smooth morphism, Preprint 2001, Universität Bielefeld.

[16] Lewis L.G., May J.P., Steinberger M., Equivariant Stable Homotopy Theory, in: Lecture Notes in Math., vol. 1213, Springer-Verlag, 1986.

[17] LodAY J.-L., Cyclic Homology, in: Grundlehren der mathematischen Wissenschaften, vol. 301, Springer-Verlag, 1992.

[18] MacLane S., Categories for the Working Mathematician, in: Graduate Texts in Mathematics, vol. 5, Springer-Verlag, 1971.

[19] Madsen I., Algebraic K-theory and traces, in: Current Developments in Mathematics, 1995, International Press, Cambridge, MA, 1996, pp. 191-321.

[20] Mandell M.A., May J.P., Equivariant orthogonal spectra and S-modules, Mem. Amer. Math. Soc. 159 (2002).

[21] Matsumura H., Commutative Ring Theory, in: Cambridge Studies in Advanced Mathematics, vol. 8, Cambridge University Press, 1986.

[22] May J.P., Simplicial Objects in Algebraic Topology, in: Chicago Lectures in Mathematics, University of Chicago Press, Chicago, IL, 1992, Reprint of the 1967 original.

[23] Mumford D., Lectures on Curves on an Algebraic Surface, in: Annals of Mathematics Studies, vol. 59, Princeton University Press, Princeton, NJ, 1966.

[24] Rognes J., Topological cyclic homology of the integers at two, J. Pure Appl. Algebra 134 (1999) 219-286.

[25] TOM DIECK T., Orbittypen und äquivariante Homologie II, Arch. Math. (Basel) 26 (1975) 650-662.

\footnotetext{
Lars HESSELHOLT

Massachusetts Institute of Technology,

Department of Mathematics, Cambridge, MA 02139, USA

E-mail: larsh@math.mit.edu
}

Ib MADSEN

Matematisk Institut,

Aarhus Universitet,

8000 Århus Denmark

E-mail: imadsen@imf.au.dk 